\title{
Progress Report No. XXIII
}

\author{
Laboratory for Insulation Research \\ Massachusetts Institute of Technology
}




\section{DISCLAIMER}

This report was prepared as an account of work sponsored by an agency of the United States Government. Neither the United States Government nor any agency Thereof, nor any of their employees, makes any warranty, express or implied, or assumes any legal liability or responsibility for the accuracy, completeness, or usefulness of any information, apparatus, product, or process disclosed, or represents that its use would not infringe privately owned rights. Reference herein to any specific commercial product, process, or service by trade name, trademark, manufacturer, or otherwise does not necessarily constitute or imply its endorsement, recommendation, or favoring by the United States Government or any agency thereof. The views and opinions of authors expressed herein do not necessarily state or reflect those of the United States Government or any agency thereof. 


\section{DISCLAIMER}

Portions of this document may be illegible in electronic image products. Images are produced from the best available original document. 
The work reported in this document was made possible through support extended to the Massachusetts Institute of Technology, Laboratory for Insulation Research, jointly by the Navy Department (Office of Naval Research), the Army Signal Corps, and the Air Force under ONR Contract Nonr-1841(10), NR-017-421; and, in part, by the Air Force under Contract AF 30(635)-2872 and the Atomic Energy Commission under Contract AT (30-1)-1937. Reproduction of this article in whole or in part is permitted for any purpose of the United States Government. 


\section{Progress Report No. XXIII}

Laboratory for Insulation Research

Massachusetts Institute of Technology

Cambridge, Massachusetts

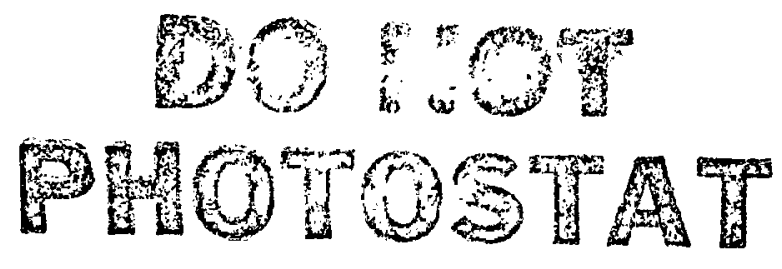

Nonr -1841 ( 10$)$

Contracts: AF 30(635)-2872

June, 1958

AT $(30-1)-1937$ 
Table of Contents

Page

Organization Chart

Publications

Survey

I. Dielectric Spectroscopy

A. Electrical Range . . . . . . . . . . . . . . . . 6

1. "Tables of Dielectric Materials, Volume VI" . . . . . . 6

2. Frequency response of ferroelectrics . . . . . . . . . . 6

3. Four-terminal measurements of $\epsilon^{*}$ at high temperatures . . 8

4. Wide-range Schering bridge . • . . . . . . . . . 9

5. Rectangular coaxial line for temperatures to $1000^{\circ} \mathrm{C}$. . . 9

6. Calculation aids for complex dielectric constant . . . . 10

7. Dielectric spectroscopy of fluids under pressure . . . . . 10

B. Optical Range . . . . . . . . . . . . . . . . . . . . 10

1. Instrumentation . . . . . . . . . . . . . . . 10

2. Line shapes . • • . . . . . . . . . . . . . . . . 11

3. Absorption spectra of titanium dioxide single crystals (rutile) from the ultraviolet to the infrared as a function of temperature . . . . . . . . . . . . .

C. Electrical and Optical Investigation of Charge-Carrier Trapping in Rutile Single Crystals . . . . . . . . . . . . . . 15

II. Magnetic Resonance

1. Instrumentation and Scope . . . . . . . . . . . . . 17

2. Magnetic resonance spectroscopy at one centimeter . . . . 17

3. Ferromagnetic resonance in magnetite . . . . . . . . . . 19

4. Resonance in garnet and ruby at $10 \mathrm{kMc}$. . . . . . . . . 20

III. High-Field-Strength Research

1. Conduction in hexane . . . . . . . . . . . . . . 21

is $\quad 3$ 
2. Photoconductivity and space-charge build-up in thallium bromo-iodide (KRS-5) • . . . . . . . . . . . .

3. Preparation of thin films of fused quartz and Vycor glass

4. Electric conductivity in glass films . . . . . . . . . . . . 24

5. Dielectric breakdown of alkali halides . . . . . . . . . 24

6. Interaction of electrons and holes with lattice ions in polar çrystals . . . . . . . . . . . . . . 25

7. Field-strength effects in cadmium sulfide single crystals 26

IV. Ferroelectrics and ferromagnetics

A. Ferroelectrics . . . . . . . . . . . . . . . 30

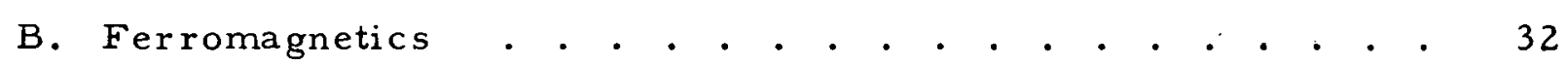

1. Magnetic properties of yttrium-iron garnet . . . . . . • 32

2. Magnetic oxides under high pressure . . . . . . . . . . 37

V. Semiconductors.

1. Optical properties of the isoelectronic compounds of germanium . . . . . . . . . . . . .

2. Optical and electrical properties of binary compounds of

$\mathrm{II}_{\mathrm{b}}$ with $\mathrm{VI}_{\mathrm{b}}$ elements $\quad \cdot \quad \cdot \quad \cdot \quad \cdot \quad \cdot \quad \cdot \quad \cdot \quad \cdot \quad \cdot \cdot$

3. Electrical conductivity of magnetite . . . . . . . . . . 43

4. Solid ionic conductors in electrochemical power sources . : 43

5. Hall-effect instrumentation . . . . . . . . . . . . . . . 46

6. Hall-effect apparatus for low-impedance samples. . . . . 47

VI. Single Crystals

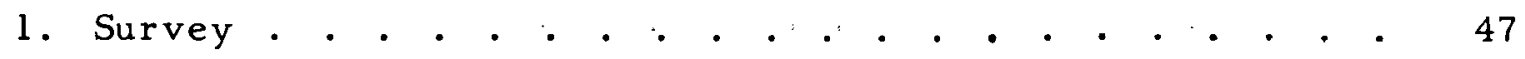

2. Purification of alkali halides by zone refining . • • . • . 48

3. Thermal etching . . . . . . . . . . . . . . . . . 48

4. Ionic conductivity of potassium bromide single crystals . . 50

5. Orientated growing of large lead single crystals . . . . $\quad$. 50 
6. Color centers in cesium halides . . . . . . . . . 52

7. Color centers in alkaline-earth fluorides . . . . . . 52

VII. Ceramics

1. High-density ceramics . . . . . . . . . . . . 53

2: High-pressure firing . . . . . . . . . . . . . . 54

3. Magnetic exchange interactions . . . . . . . . . 54

4. Modified atomizingmburner operation . . . . . . . 54 


\section{ORGANIZATION CHART}

\section{LABORATORY FOR INSULATION RESEARCH}

June 1, 1958

Professor A. R. von Hippel, Director

Dr. Alexander Smakula, Associate Director

S. M. Kingsbury, Business Manager

DIELECTRIC MEASUREMENTS

William B. Westphal, Group Leader

Barbara B. East

Benjamin R. Moon

Vytautas Klemas, Grad. Student, E.E.

SPECTROSCOPY

Dr. Robert D. Waldron, Group Leader

Bernard H. Soffer, S. M., Grad. Student, E.E.

MAGNETIC SPECTROSCOPY

Prof. Perry A. Miles, Group Leader

James S. Hyde, Grad. Student, Physics

Terence E. McEnally, Jr., M.S., Grad. Student, Physics HIGH VOL TAGE

Dean A. Powers, E.E., Group Leader

Carl W. Nelson

Dr. Marco Bono

Lloyd R. Schissler, Grad. Student, E.E.

Stephen J. Nettel, Grad. Student, Physics

Krishna G. Srivastava, M.S.

Harry Gildea, M.S., Grad. Student, E.E.

Ralph Moon, M.S., Grad. Student, Physics

\section{FERROMAGNETICS}

Prof. David J. Epstein, Group Leader

Boguslaw Frackiewicz

Theodore P. Janu'sz, M.A., Grad. Student, Physics

Thomas W. Stewart

FERROELECTRICS

Dr. Peter W. Forsbergh, Group Leader

SEMICONDUCTORS

Dr. David S. Tannhauser

Dr. John Mrgudich

William A. Navipour, Grad. Student, E.E.

George B. Wright, Grad. Student, Physics

Roland Coelho, M.Sc., Grad. Student, Physics
CRYSTAL GROWTH

Dr. Alexander Smakula, Group Leader

Peter Avakian, Grad. Student, Physics

William J: Scouler, Grad. Student, Physics

Bernard J. Wuensch, S. M., Grad. Student, Physics

Carl F. W. Ekman, Grad. Student, Chemistry

John C. Gilland

HIGH PRESSURE

Dr. Peter W. Forsbergh, Jr.

Eldred B. Littlefield, Grad. Student, Physics

CERAMICS

Prof: George Economos, Group Leader

John S. Waugh, M.S., Grad. Student, Metallurgy

Thomas R. Clevenger, Jr., Grad. Student,

INORGANIC CHEMISTRY

Metallurgy

Janis Kalnajs, Dipl. Eng. Chem.

ORGANIC CHEMISTRY

Dr. Laurence G. Wesson

EDITOR

Joseph Stein

LIBRARIAN

Aina Sils

SECRETARY

Anna $F$. Crice

DRAFTSMAN

John J. Mara

MACHINE SHOP

Peter P. Kelleher., Foreman 
List of Publications, December 1, 1957 to June 1, 1958

$\underline{\text { Reports }}$

Progress R'eport No. XXII, December, 1957.

Technical Report 125 "Space-Charge Build-Up in Potassium Bromide Crystals by A-C Methods;" by D. K. Donald.

Technical Report 126 "Tables of Dielectric Materials, Vol. VI, "by B. B. East and W. B. Westphal.

Technical Report 127 "Electrical and Optical Investigation of ChargeCarrier Trapping in Rutile Single Crystals," by K. G. Srivastava.

Interim Quarterly Letter Report No. 8, August 15, 1957 to December 31, 1957, Rome Air Development Center, Griffiss Air Force Base, Contract No. AF 30(635)-2872, "Ferromagnetic Semiconductors as Therrrosensitive Devices," by D. J. Epstein.

\section{Published Papers}

"An Improved X-Ray Method for Determining Cation Distribution in Ferrites," L. P. Skolnick, S. Kondo, and L. R. Lavine, J. Appl. Phys. 29, 198 (1958).

"Answers to Sputnik?" A. von Hippel, Bull. Atomic Scientists 14, 115 (1958); Tech. Rev. 60, 251 (1958).

"Discrete States for Singular Potential Problems," F. L. Scarf, Phys. Rev. $109,2170(1958)$.

\section{Papers Presented at Technical Meetings}

"Evaluation of Some Methods of Nickel Ferrite Preparation," G. Economos, Annual Meeting of American Ceramic Society, April 27-30, Pittsburgh, Pa. 


\section{Progress Report No. XXIII}

on

Contracts Nonr-1841(10); AF 30(635)-2872 and AT (30-1)-1937

\section{SURVEY}

Publication of "Molecular Science and Molecular Engineering" this fall (The Technology Press of M.I.T. and John Wiley and Sons) terminates one major task of the Laboratory for Insulation Research: to help create a synthesis of knowledge and a true alliance between science and engineering.

"The book* $\dagger$ begins with an orienting look at the procedures of classical science and engineering (Chap. 1). Here we find axiomatic facts and their quantitative formulation, matter classified by macroscopic criteria, materials described by numerical coefficients of response to external probing. Mathematical logic takes over and covers this framework with a theory of streamlined elegance; inquiry gives way to application until the horizon proves too limited, and new adventure begins. In our specific discussion. we refer to electrical science and engineering because questions about the electrical structure of matter lead to the particles of the molecular world.

Chapter 2 introduces these particles: first the atoms with their electron clouds of standing-wave modes described by quantum mechanics, the response of atoms to electric and magnetic fields by Stark and Zeeman effects, the gyroscopic nature of magnetic moments. A dilemma comes into focus with the build-up scheme of the periodic system using hydrogenlike

* This quotation is from "Molecular Science and Molecular Engineering." Copyright (C) 1958, Massachusetts Institute of Technology.

t The authors: Chaps. 1,2,3,9,15, and 21, A. von Hippel; Chap. 4, J. P. Kuettner; Chap. 5, S. C. Brown; Chap. 6, E. O. Johnson; Chap. 7, B. Lewis; Chap. 8, O.K. Mawardi and A. von Hippel; Chap. 10, C. G. Swain; Chap. 11, W. H. Stockmayer; Chap. 12, A. Smakula; Chap. 13, R. Smoluchowski; Chap. 14, E. Orowan; Chap. 16, P. W. Forsbergh, jr.; Chap. 17, W. P. Mason; Chap. 18, D. J. Epstein; Chap. 19, R. A. Ramey, Jr., and B. W. Lovell; Chap. 20, J. W. Mayer and D. O. Smith; Chap. 22, R. M. Fuoss; Chap. 23 C. D. Coryell and Y. Marcus; Chap. 24, R. B. Adler; and Chap. 25, K. Martinez. 
wave functions: Quantum mechanics has to deal with strong interactions between particles and, like classical physics, can handle its multibody problems only by approximation. This fact, added to the principal limitation imposed on observability by the quantum of action, requires critical examination of any mathematical solution as to its actual range of validity. The remainder of Chap. 2 sketches the interaction of atoms: binding and repulsion effects, release or attachment of electrons, various bond types, the stereostructure and isomerism of molecules, permanent electric dipole moments, and the radii of atoms and ions.

This acquaintance with atoms and molecules suffices for a discussion of the electrical behavior of gases (Chap. 3). A gas at normal temperature should be an ideal insulator. The observation of conduction at low field strength therefore leads to exciting discoveries concerning the initiating charge carriers and their effects. In stronger fields, charge-carrier multiplication sets in as described by Townsend's avalanches. Breakdown itself requires a feedback mechanism causing instability; a variety of effects may contribute to the sudden collapse of the gas insulation. Spacecharge formation contracts the field into a cathode fall; electrons are liberated from the cathode by ion bombardment, photoeffect, the impact of radicals, or by field emission; short-ranging ultraviolet radiation may ionize the gas as an avalanche develops, and so on. Visual evidence is needed to interpret the electrical data. Even the simple turning of a camera provides useful information about the mechanism of sparks and lightning strokes; Kerr cell shutters allow photographing momentary development stages; and Lichtenberg figures show in most beautiful detail the unfolding of events. The Wilson cloud chamber and, finally, direct recording succeeded in measuring individual avalanches and their progeny. 
"With this preview, the stage is set for the inquiry of Chap. 4 concerning our present understanding of thunderstorms and the electrical state of the atmosphere. This is an age-old problem, with ever new ramifications, in contrast to a very recent field, the microwave discharges. Here, as Chap. 5 reports, everything is simplified and well understood: Charge generation in the gas balances diffusion, the walls of the container are limiting boundaries instead of electrodes, and a quantitative theory finds quantitative confirmation.

In Chap. 6, the theme of gas discharges is handed over to the molecular engineer for a review of present applications and future trends. Chapter 7 turns from electrical action to chemical reaction in gases, especially to processes leading by cumulative acceleration to explosion.' This crescendo ends our preoccupation with gases.

Chapter 8 broadens the basis for a general understanding of all states of aggregation. Thermodynamic systems, their macroscopic treatment and statistical interpretation, come into focus. The idealized models of the perfect gas, the perfect elcctron gas and the perfect phonon gas, leading to Maxwell-Boltzmann, Fermi-Dirac, and Bose-Einstein statistics, are contrasted to the perfect crystal embodying complete order and to the imperfect gas leading to phase equilibria. The problem of nucleation arises; and the extension of thermodynamics to systems containing electric and magnetic energy is indicated. This long-distance view is complemented in Chap. 9 by a close-up inquiry about the actual building laws for condensed phases. In sequence there appear the results of macroscopic and molecular crystallography; the concepts of dense packing, radius, ratio, and interstitial position in their application to a variety of crystal structures; the transition from finite to infinite molecules in lattices; the building of structures from tetra- 
hedral and octahedral groups; polymers and networks showing all gradations between short-rarge and long-range order; and the imperfections inherent in ordered systems.

In the subsequent five chapters a fill-in and real mixing of diversified knowledge takes place. First the chemist speaks about our present insight into successive steps of reaction in liquid systems (Chap. 10). Then the physical chemist takes over in a review of polymer formation (Chap. 11), followed by the physicist with a detailed report on crystal growth, the techniques used and results achieved (Chap. 12). After this account of the formation of structures, the discussion shifts to disturbances of structure caused in solids by irradiation (Chap. 13) and under mechanical stress (Chap. 14). These five chapters survey a Disneyland of molecular action: From molecules turning inside out like umbrellas to nuclear shooting galleries and to dislocation mills spinning crystals to destruction. The reader should not despair if it takes time to visualize this molecular spectacle.

With Chap. 15 we return to the world of electric and magnetic phenomena. Electric and magnetic dipoles are considered in parallel presentation: First their individual contribution to polarization and magnetization, for static fields and in resonance or relaxation response; then coupled dipole systems come under scrutiny. Short-range energy terms prove decisive, and not long-range dipole forces, in producing piezoelectrics, ferroelectrics, ferromagnetics and their antipodes. The principles that make their behavior understandable are discussed; with this background secured, the attention turns to presentday ferroelectric and ferromagnetic materials and devices (Chaps. 16 to 19). Here the molecular scientist, building crystal structures, and the molecular engineer, searching for new applications, come into their own. Chapter 20 finishes the dipole story by presenting the presently most provocative devices: 
the masers, parametric amplifiers and thin-film magnetic memories, and by giving some speculative implications for the future.

Once more the spotlight swings to a new subject: The action of charge carriers in liquids and solids. A surveying section (Chap. 21) provides general briefing on charge-carrier stabilization in condensed systems; on ions, their surroundings and motion in liquids and crystals; on electrons in crystals and their behavior under the rules of quantum mechanics. Chapters 22 to 24 follow up with subjects of special interest. The first two sections discuss ions in peculiar and related situations: as polyelectrolytes, i.e., galaxies of cations or anions appended to polymer molecules and immersed in solutions of compensating countercharges; and as ion exchange resins, which are actually polyelectrolytes frozen into a plastic framework. Chapter 24 analyzes the action of the presently most important and best understood semiconductor devices: rectifier diodes and transistors.

A short closing chapter focuses attention on the challenges of this space age and calls molecular engineering to the rescue."

If possible, the Laboratory should now retreat behind a "Dew Line" and supported by long-range funds for fundamental research concentrate on challenging ventures: studies on high field strength phenomena; on the creation of more nearly ideal crystals, films and surface structures; on the coupling of electric and of magnetic dipole systems; on the structure and behavior of ions in electrolytes; and on the mobilization and trapping of electrons and holes in solids. This progress report reflects some of our efforts along such lines.

A. von Hippel 


\section{DIELECTRIC SPECTROSCOPY}

\section{A. Electrical Range}

1, "Tables of Dielectric Materials, Volume VI"' (B. B.. East and W. B. We stphal)

This volume of the Tables (Tech. Rep. 126) is supplementary to Volumes IV and V. It includes new materials together with extended data on some of the materials listed in previous Tables. Frequency runs are given in graphical instead of the tabular form used in Volumes III to V.

In addition to materials mentioned in previous progress reports, we are including two Teflon materials:

"Duroid" 5650 . Teflon plus ceramic fibers. Rogers Corp.

"Duroid". 5850 Teflon plus fiberglass . Rogers Corp.

2. Frequency response of ferroelectrics (W. B. Westphal and I. S. Schwartz)

A coaxial re-entrant cavity (Fig. 1) for measuring smali plates of high

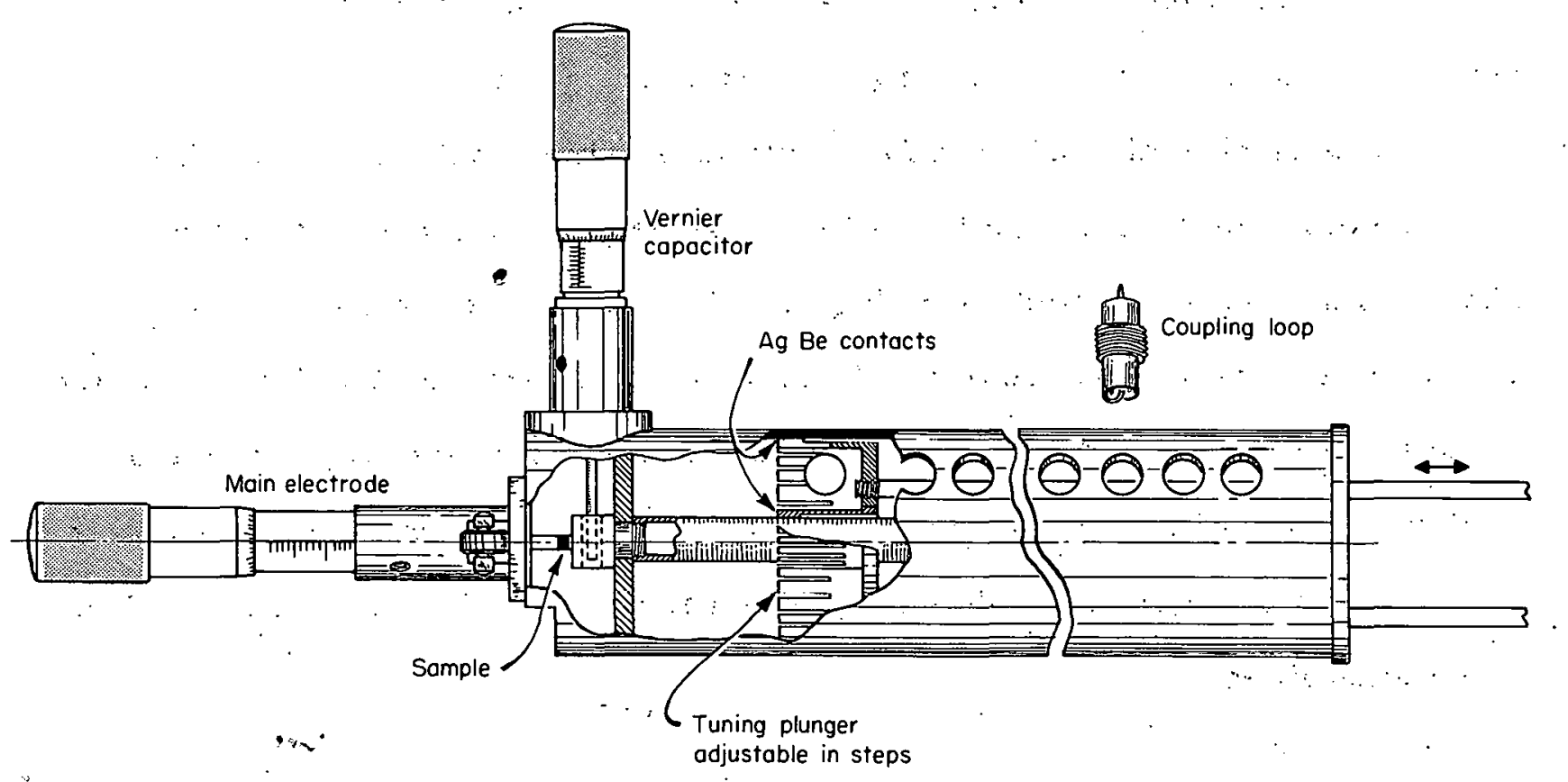

Fig. 1. Re-entrant cavity for measuring small plates of ferroelectrics in the 250 to $1000 \mathrm{Mc}$ range. 
dielectric constant ceramics or crystals has been completed for use in the frequency range 250 to $1000 \mathrm{Mc}$. The cavity length is variable in steps of $2 \mathrm{~cm}$; a small linear vernier capacitor is used to obtain the width of the resonant curve.

A small shielded microstrip standing-wave detector (Fig. 2) has been constructed for measurements on the same samples in the range 750 to

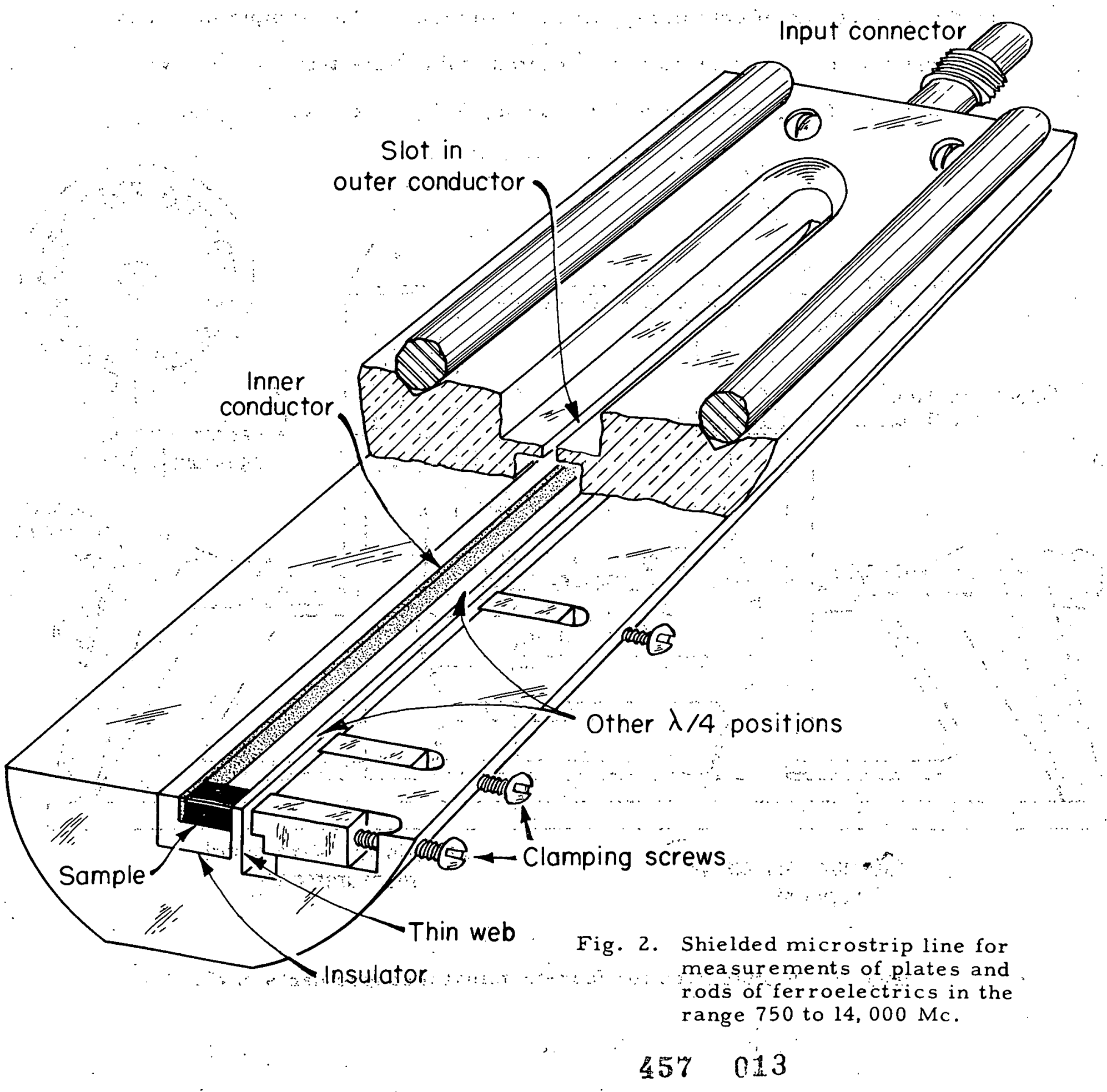


$14,000 \mathrm{Mc}$. One section of the outer conductor (Fig. 2) is thin so that the samples may be clamped or soldered to the conductors at regions of high electric field strength for selected measuring frequencies.

3. Four-terminal measurements of $\epsilon^{*}$ at high temperatures (W. B. Westphal)

A slotted oven (Fig. 3) for temperatures up to $1200^{\circ} \mathrm{C}$ will aid in the study of electrode effects in sapphire and aluminas. The sample mounting arrangement has short leads and no heated solid dielectrics in shunt with the sample. A high impedance $7 \times 10^{8}$ ohms, $0.5 \mu \mu f d$ at $1 \mathrm{kc}$ ) detector unit

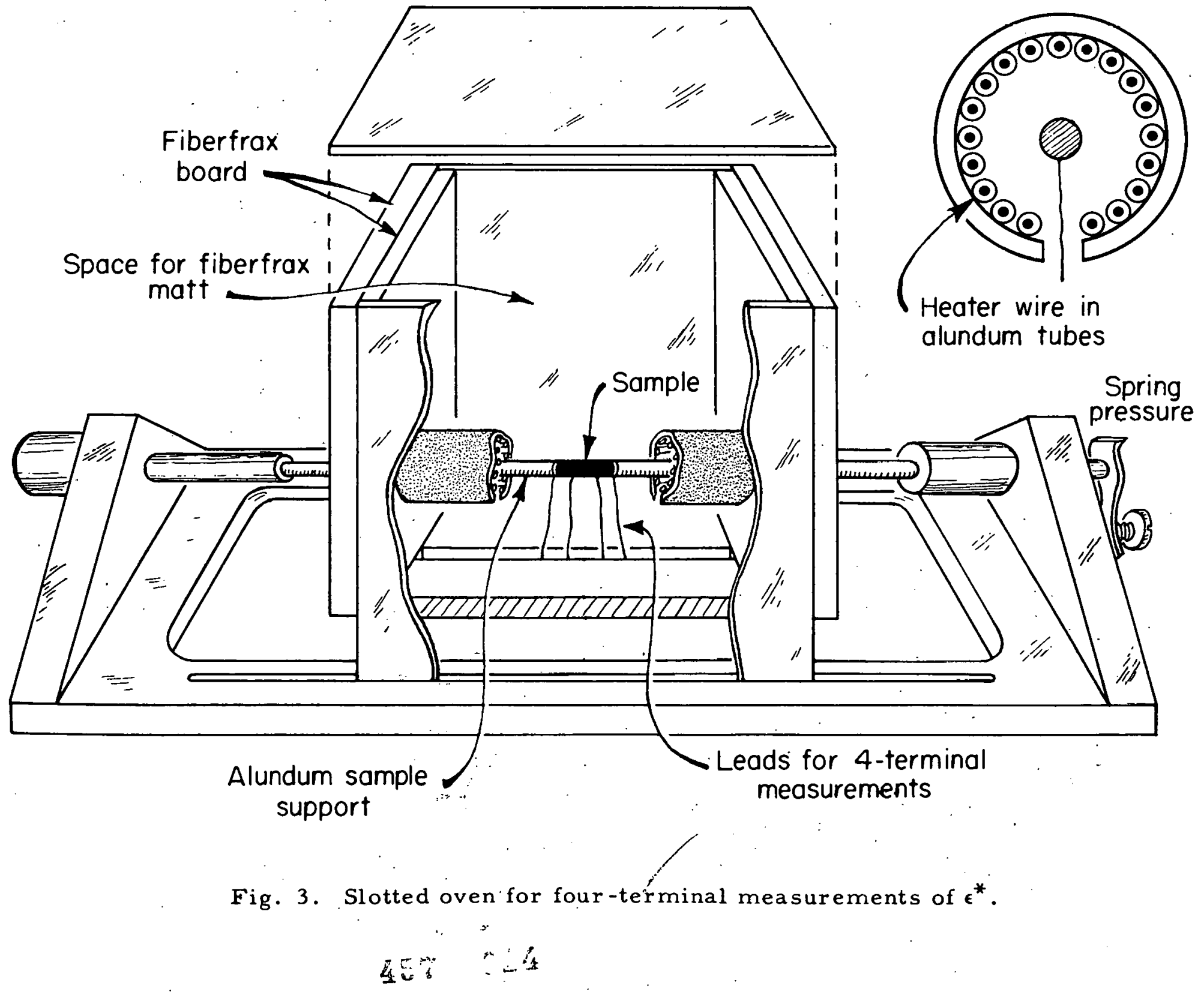




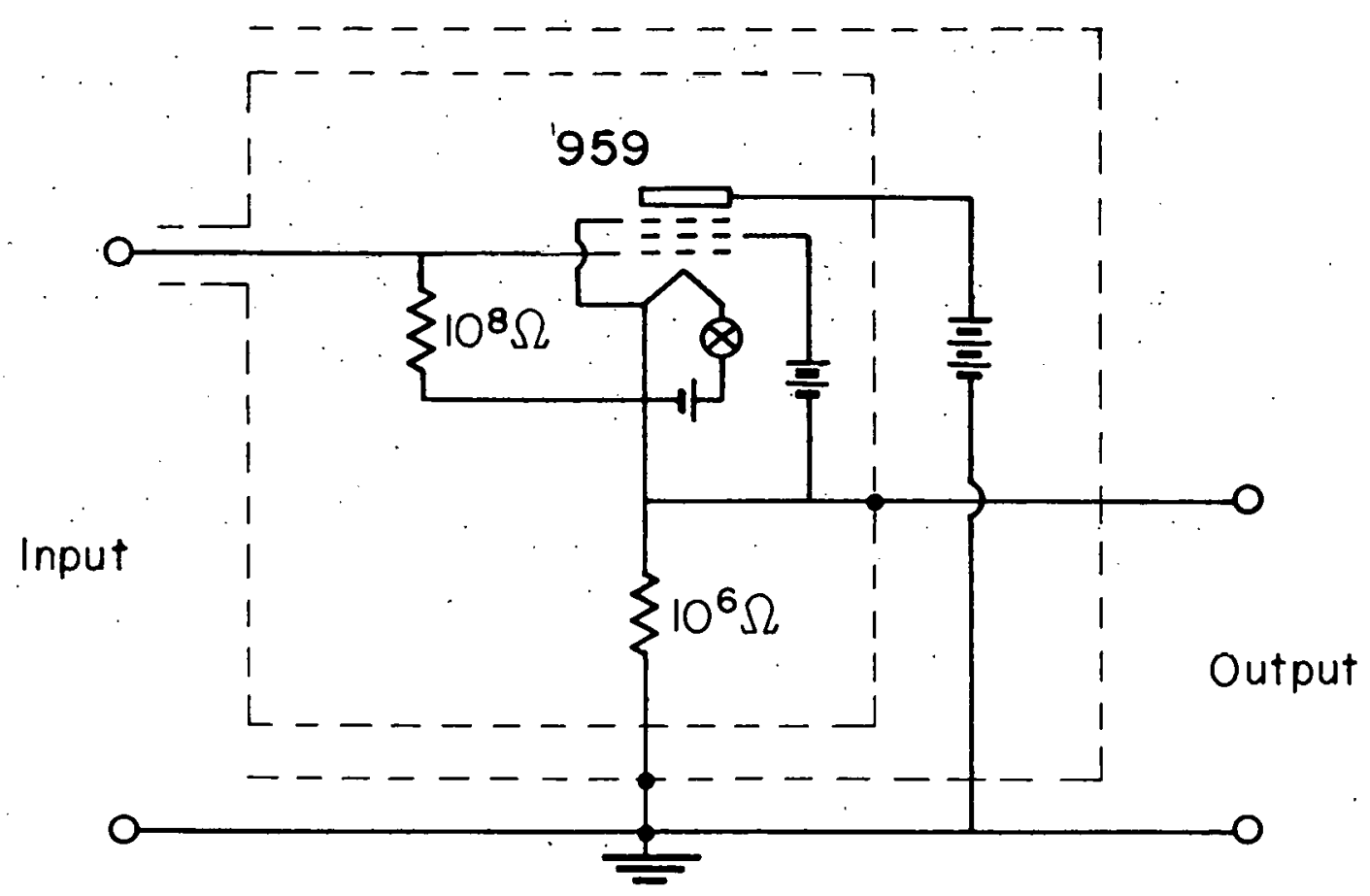

Fig. 4. Input unit for detector in four-terminal measurements of the complex dielectric constant.

(Fig. 4) has been constructed for use in a four-terminal measuring circuit.

\section{Wide-range Schering bridge (B. Moon)}

One unit of the bridge, the oscillator, has been completed except for the motor drive. The frequency range is $1 \mathrm{cy}$ to $10 \mathrm{Mc}$ in 14 bands. The accuracy of the output frequencies is better than 0.5 percent over most of the frequency range. The frequency selective portion of the self-tracking detector is now being constructed.

5: Rectangular coaxial line for temperatures to $1000^{\circ} \mathrm{C}$ (W. B. Westphal) Materials have been ordered for a coaxial line with Inconel sample holders to operate in the frequency range 1000 to $3000 \mathrm{Mc}$. The crosssection geometry (Fig. 5) allows the application of external pressure for maintaining good contact between sample and conductors with changes in temperature. 


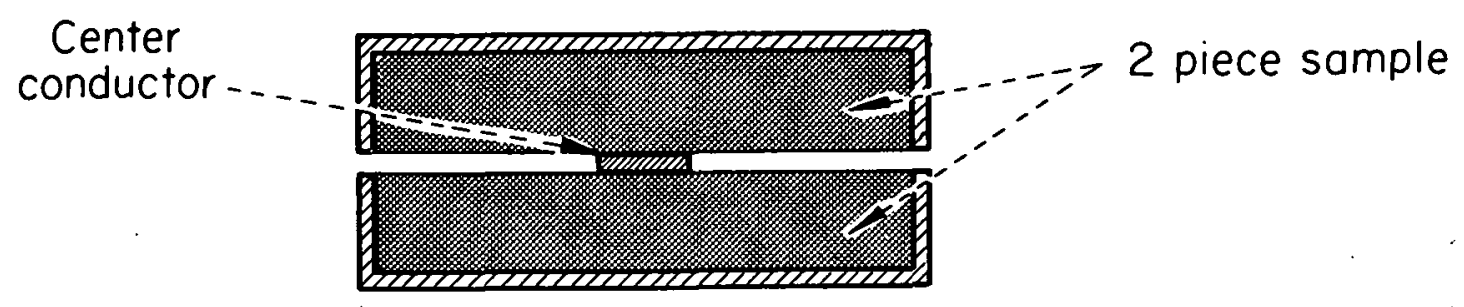

Fig. 5. Cross section of rectangular coaxial line for high temperatures.

6. Calculation aids for complex dielectric constant (B. B. East)

Tables have been prepared for use with the Central Research Laboratories' Dielectrometer at $8.5 \mathrm{kMc}$ by which one obtains the dielectric constant (for a narrow range of sample lengths) directly from the node position for samples placed in contact with a shorting disk or a quarter wavelength in front of it. The tables also give the multiplying factor for calculating $\tan \delta$ from the width of the minimum. The charts of the complex function $(\operatorname{coth} \mathbf{x}) / \mathbf{x}$ of Technical Report 36 have been expanded for increased accuracy.

7. Dielectric spectroscopy of fluids under pressure (E. B. Littlefield and P. W. Forsbergh, Jr.)

The microwave dielectric cell for high pressure has been constructed and is being tested. A new pressure cell for radio frequencies has been constructed. It is designed for 5000 atmospheres and will soon be tested. A pressure intensifier with integral bulk-modulus pressure gauge has been designed and is being constructed.

\section{B. Optical Range}

1. Instrumentation (R. D. Waldron)

The modifications of the Beckman IR -3 spectrophotometer for far infrared operation are essentially complete. A new Golay detector with diamond $357 \cdot r-16$ 
window has been installed and tested. Operation in higher order (near 45 microns) has been achieved, but some additional filtering is necessary to reduce stray light for longer wavelengths.

An auxiliary mirror system to permit double-pass operation on each half of the double monochromator has been designed and partially assembled. The reversing mirrors were curved to compensate for additional slit image curvature.

The external optics were modified to provide for an external focus prior to the entrance slit. This facilitates work with high-temperature cells and in reflection because a smaller cross section is required to fill the aperture of the monochromator.

\section{Line shapes (R. D. Waldron)}

A theoretical study of the dependence on intensity and bandwidth of spectra on various absorption parameters (Progress Report No. XX, p. 34) has been completed and will appear shortly as a technical report. Earlier mathematical difficulties with the equalions for reflectivity have been avoided by evaluation of an auxiliary function in the complex plane. Explicit relations for the frequency shift as a function of intensity and damping of the maxima for the parameters: loss factor, conductivity, loss tangent, absorption indices $k$ and $k=n \kappa$, absorption coefficient, and reflectivity have been obtained, and graphical aids developed for evaluating such shifts. Figure 6 shows the frequency shift of the maximum reflectivity as a function of intensity and bandwidth for a limiting refractive index of unity at the high-frequency side.

3. Absorption spectra of titanium dioxide single crystals (rutile) from the ultraviolet to the infrared as a function of temperature (B. H. Soffer)

The optical absorption of single crystals of synthetic rutile were investigated in the spectral range from 1000 to $25,000 \mathrm{~cm}^{-1}$ and $\mathrm{from}$ room 


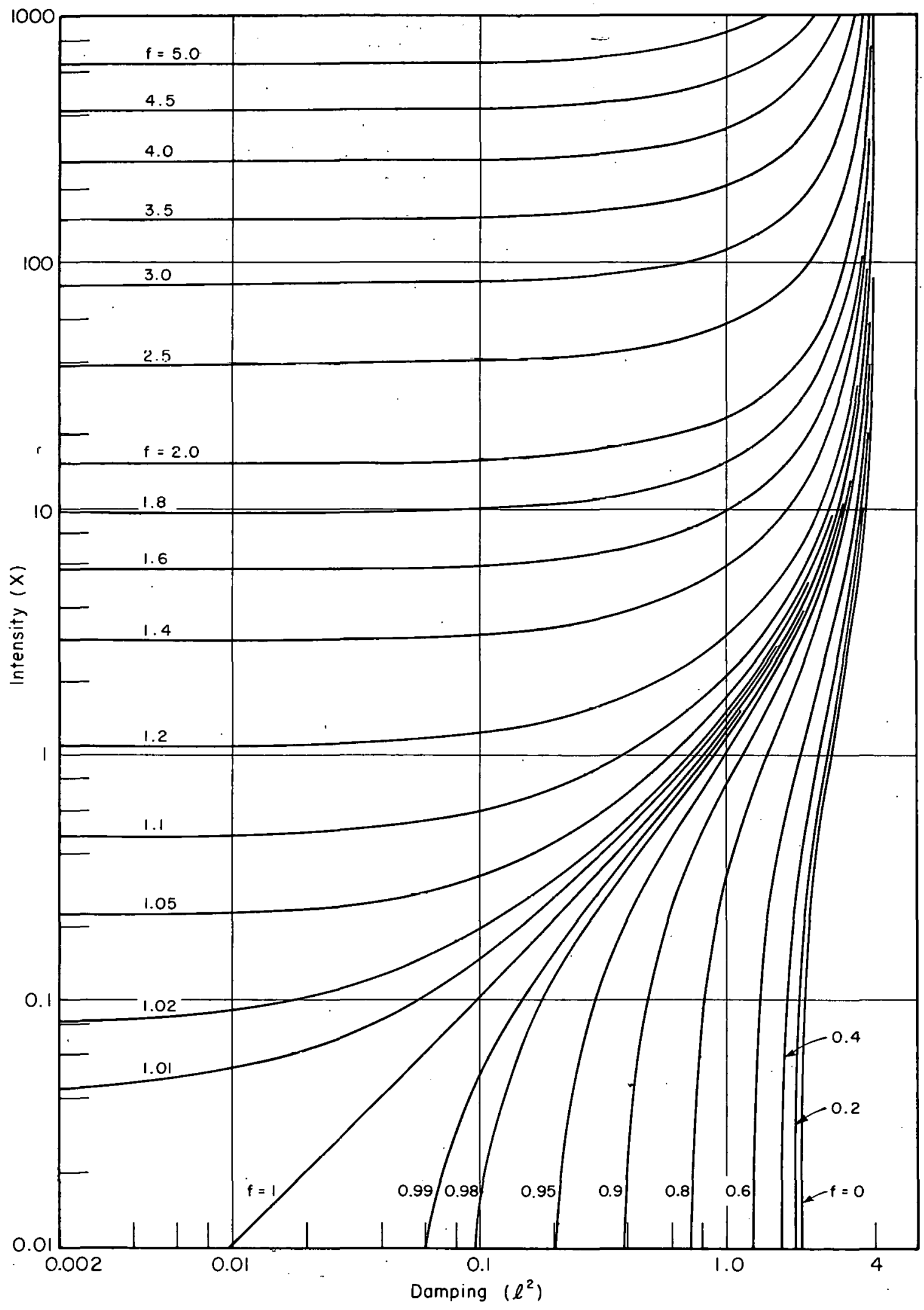

Fig. 6. Frequency dependence of the maximum of the reflectivity $R$ on intensity $X$ and bandwidth $\ell^{2}$ of a damped harmonic oscillator for $k=1(\omega \rightarrow \infty)$. 
temperature to $1000^{\circ} \mathrm{C}$.

The electronic absorption edge shifts toward lower energies at a rate depending upon the absorption coefficient. At a decadic absorption coefficient of $400 \mathrm{~cm}^{-1}$ the rate is $7.1 \times 10^{-4} \mathrm{ev} /{ }^{\circ} \mathrm{K}\left(\mathrm{Fig}\right.$. 7), while at $1 \mathrm{~cm}^{-1}$ it is $9.5 \times 10^{-4} \mathrm{ev} /{ }^{\circ} \mathrm{K}$. The broad band in the region $6850 \mathrm{~cm}^{-1}$, which occurs in reduced rutile, does not appear at high temperatures in fully oxidized rutile.

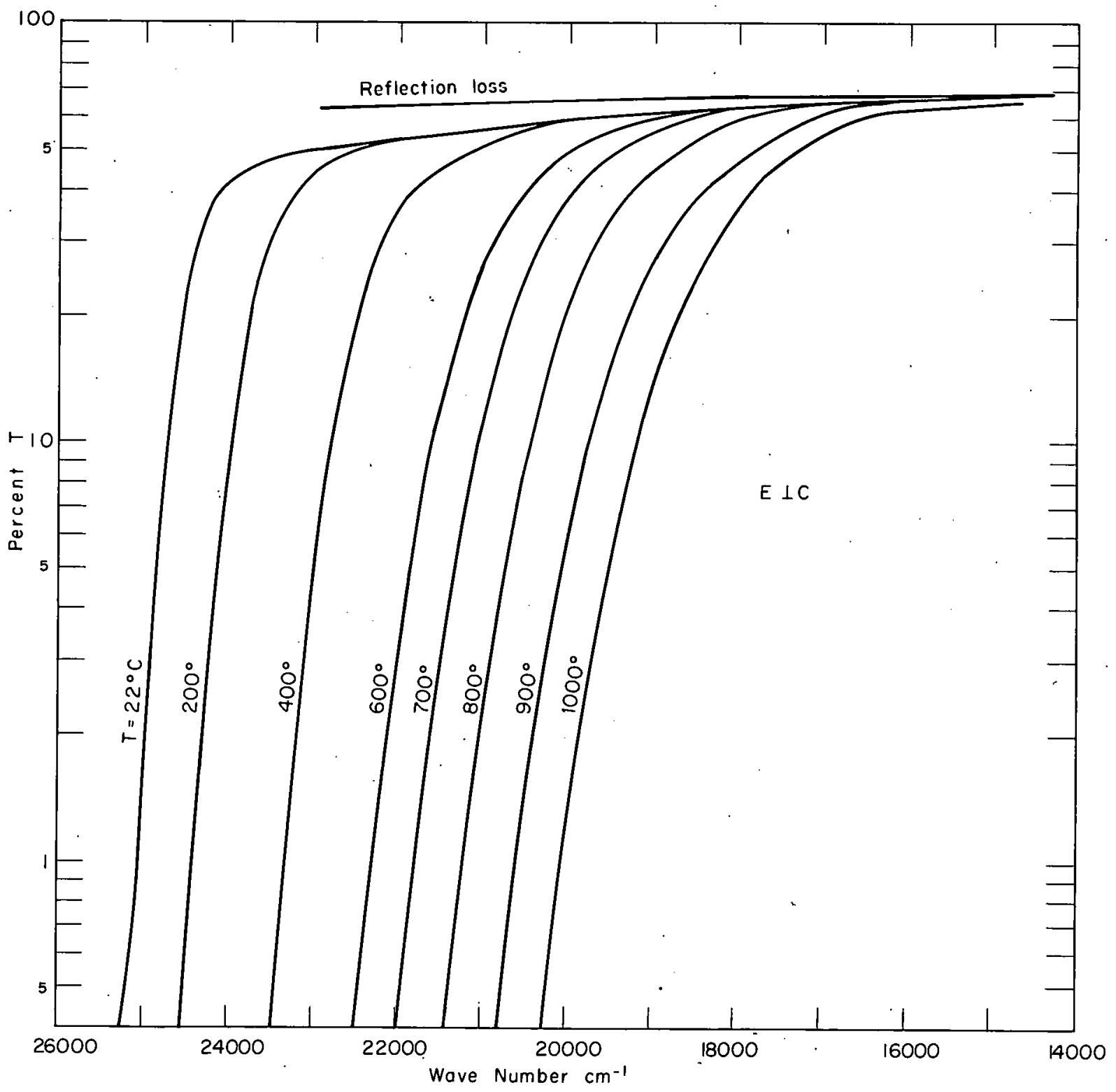

Fig. 7. Absorption spectrum of rutile sample, $29.5 \mu$ thick, as function of temperature. 
No additional absorptions at high temperatures could be detected.

A new band was discovered in the $3300 \mathrm{~cm}^{-1}$ region which was identified as being produced by $\mathrm{O}-\mathrm{H}$ valence vibrations. The band was unusually sharp and shifted with peaks at 3277 and $3322 \mathrm{~cm}^{-1}$ of half widths 28 and $13 \mathrm{~cm}^{-1}$, respectively (Fig. 8). A satellite structure of combination and

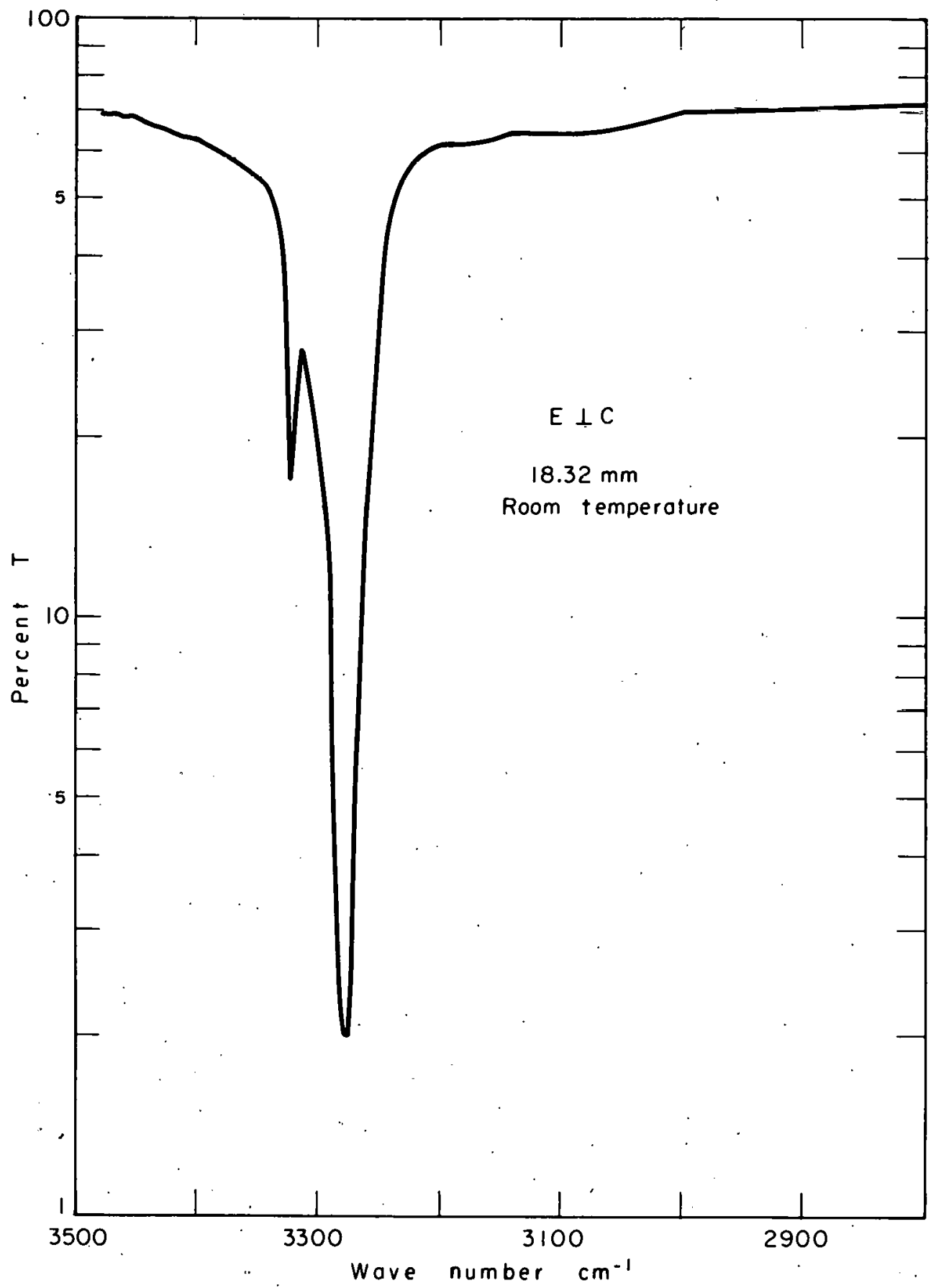

Fig. 8. Infrared spectrum of rutile in the region of $3300 \mathrm{~cm}^{-1}$.

$$
48600
$$


difference bands was also detected. Deuterium substitution revealed a band at $2442 \mathrm{~cm}^{-1}$. The entire structure showed a markedly different absorption for different light polarization.

C. Electrical and Optical Investigation of Charge-Carrier Trapping in Rutile Single Crystals (K. G. Srivastava)

Low-frequency absorption centers, observed by electrical measurements in single crystals of rutile, can be produced by thermal quenching or optical irradiation in the edge of the eigenabsorption region (4200A) (Fig. 9):

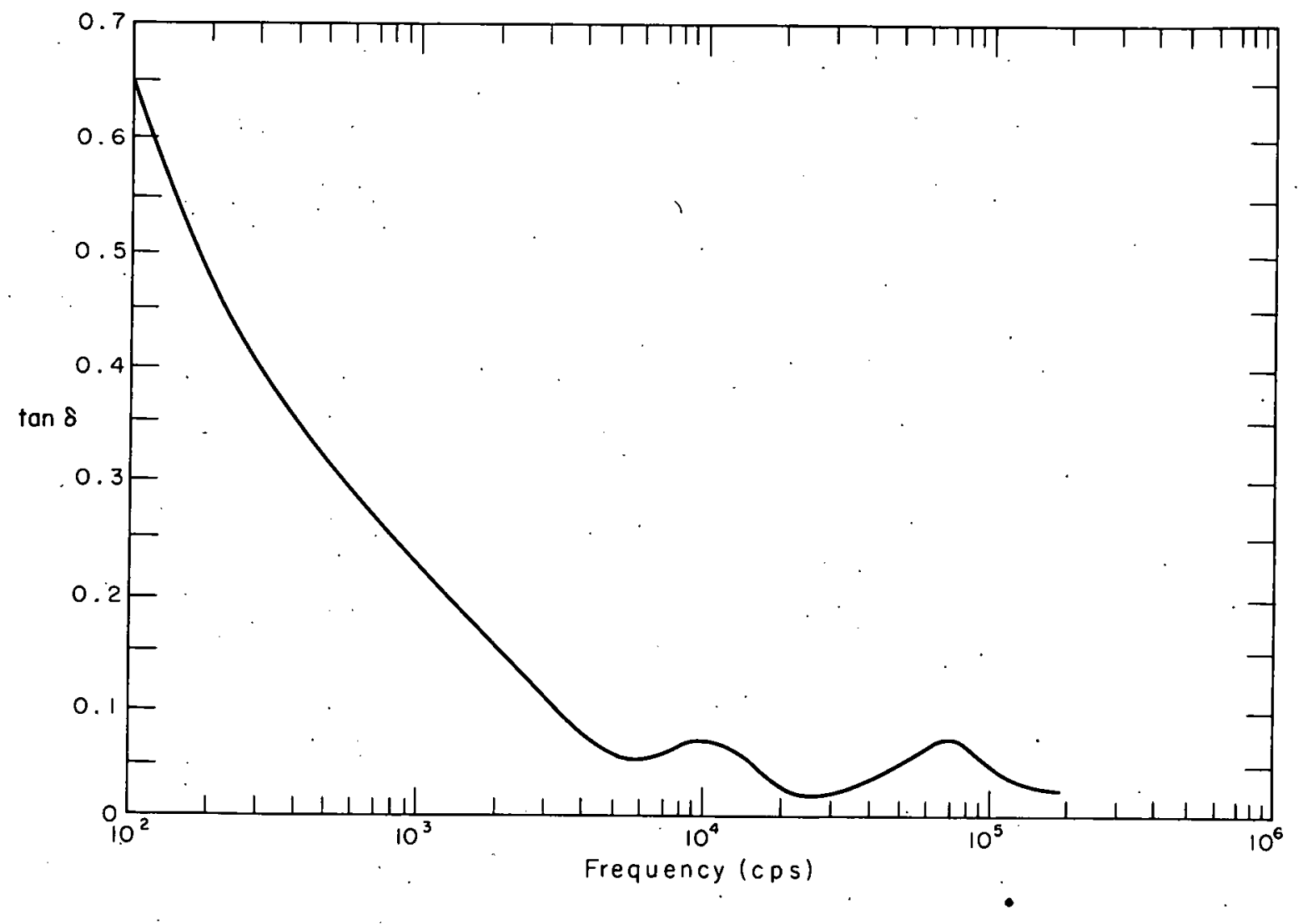

Fig. 9. Dielectric loss characteristic for rutile after long exposure to irradiation at $4200 \pm 100 \mathrm{~A}$. 


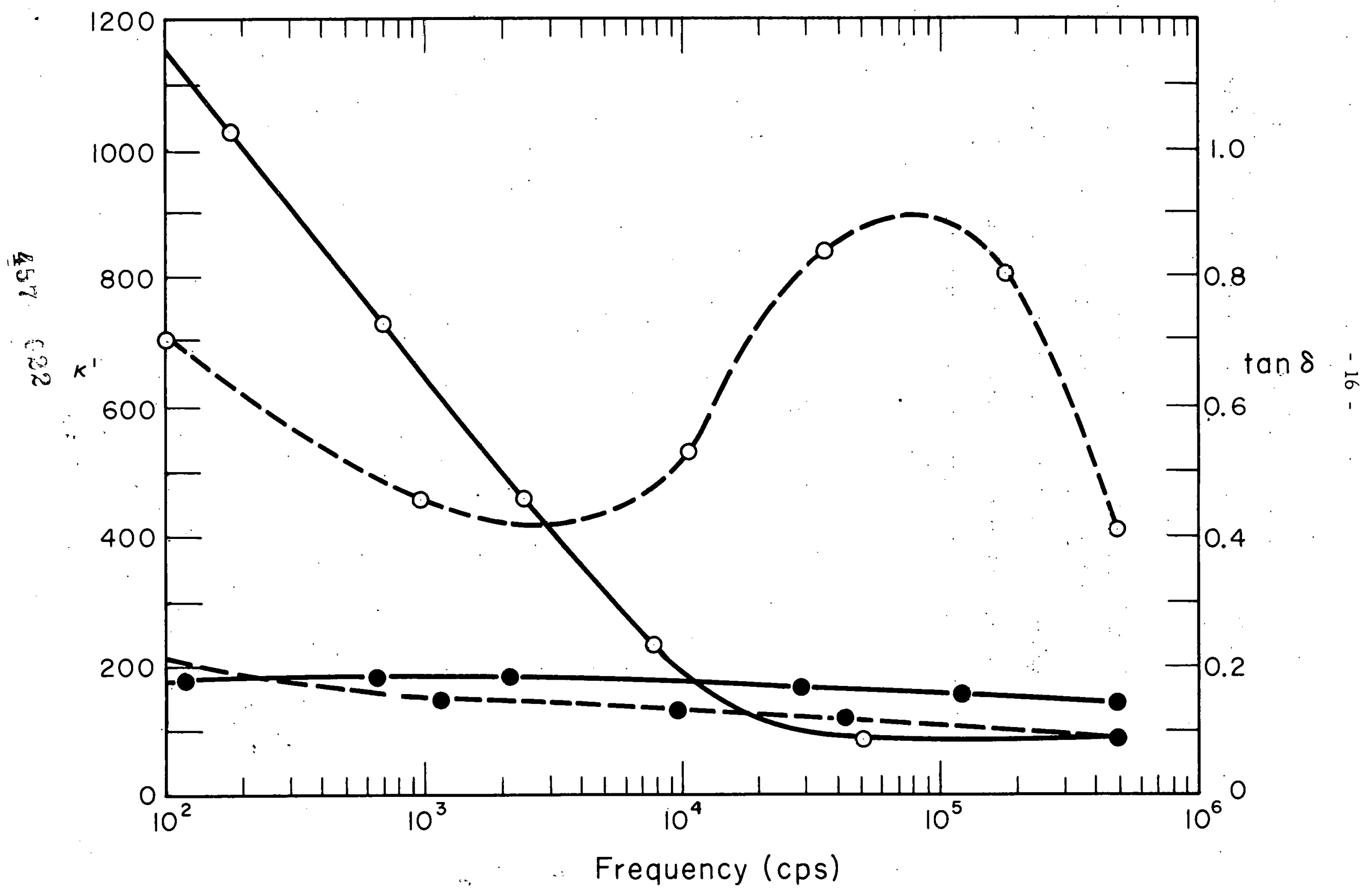

Fig. 10. Dielectric constant (solid lines) and loss (dashed lines) vs. frequency far quenched rutile sample, parallel (circles) and perpendicular (triangles) to the optic axis at room temperature. 
They are observed when electric fields are applied parallel to the optic axis crystallographic c axis) and are not visible with the field perpendicular to this axis (Fig. 10). The temperature dependence of the principal dispersion indicates an activation energy of $0: 18 \mathrm{ev}$. The centers can be explained by a localized electron transfer process along the optic axis. The introduction. of silver and copper by thermal diffusion did not produce low-frequency absorption spectra.

\section{MATINETIC RESORJANCE}

1. Instrumentation and scope (P. A. Miles, J. S. Hyde, and T. E. McEnally) Major improvement and extension of our magnetic resonance equipment has taken place in the last few months. The 24-kMc spectrometer has been increased significantly in sensitivity and dependability. A new 10-kMc instrument has been built for work at high microwave-power levels and to provide a more flexible cavity design for future measurements at high temperatures.

Currently we are working on the magnetic response of imperfections in àlkali halides and on spectra in synthetic ruby, ferrimagnetic garnets and magnetite at low temperatures.

2." Magnetic resonance spectroscopy at one centimeter (J. S. Hyde)

We have reached a sensitivity corresponding to the detection of ca. $6 \times 10^{10}$ electron spins at room temperature at a line width normalized to one gauss, on a sample of $\mathrm{KBr}$ containing $1.4 \times 10^{17} \mathrm{~F}$ centers per $\mathrm{cm}^{3}$. The Smakula formula was used to determine this concentration (Fig. 11).

The slight change in the noise level in this curve is attributed to bridge drift. The bridge was rebalanced at $R$ and the noise on the portion of the trace to the right is thought to be the lowest level attainable. The 


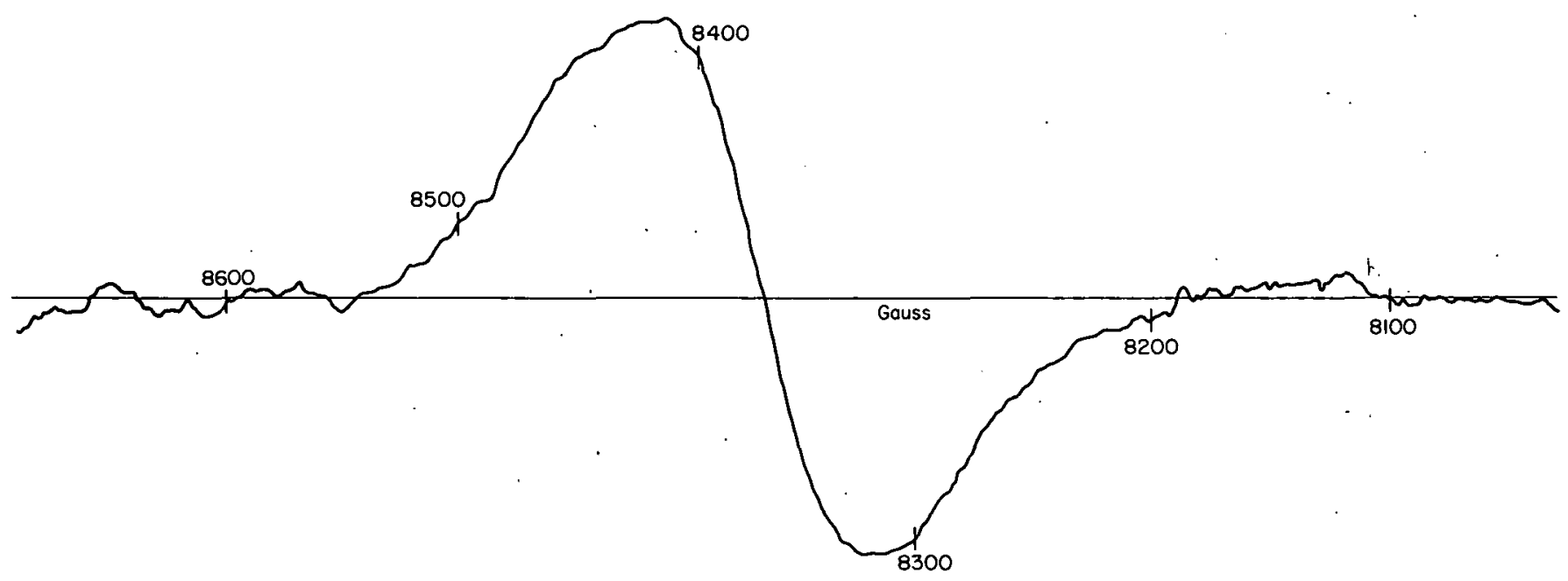

Fig. 11. F-center resonance in $\mathrm{KBr}$ (derivative of absorption).

major increase in sensitivity resulted from a critical adjustment of the Pound frequency stabilizer. Better mechanical behavior was obtained by shock mounts and use of a telescoping phase shifter. An equal arm arrangement of the stabilizer bridge $\mathrm{e}^{\mathrm{l})}$ was adopted.

Dispersion curves are about ten times as noisy as absorption curves, suggesting that klystron-frequency variation or mechanical vibration of the microwave bridge limits the sensitivity. If one attempts to eliminate the effect of klystron-frequency variations on the absorption curves by increasing the amount of amplitude out-of-balance, the noise increases, presumably because klystron power-level fluctuations begin to contribute to the noise.

The F-center resonance in $\mathrm{KBr}$ had a width of 152 gauss and occưred at a magnetic field corresponding to a $g$ value of 1.980 .

We observed the resonance of $\mathrm{Mn}^{2+}$ obtained by dissolving $\mathrm{MnCl}_{2}$ in

1) W. G. Tuller, W. C. Galloway, and F. P. Zaffarano, Proc. Inst. Radio Engrs. 36, 794 (1948). 


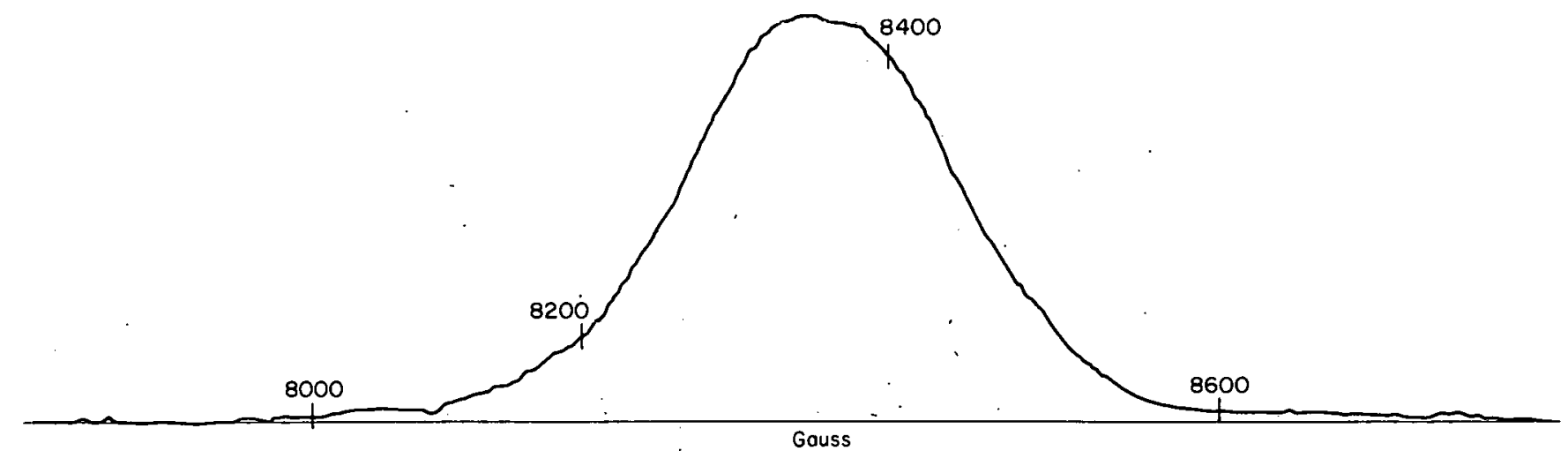

Fig. 12. Fast-passage F-center resonance in LiF.

alcohol. The $Q$ of the cavity was lowered somewhat by.residual water in the sample, but a normalized room-temperature sensitivity of $7 \times 10^{11}$ spins was obtained. The separation of the hyperfine lines is described by a nuclear spin Hamiltonian of the form $\mathcal{H}=g \beta H I+A I \cdot S$. The interaction constant A was found to be $0.0092 \mathrm{~cm}^{-1}$, in general agreement with the values found for $\mathrm{Mn}^{2+}$ diluted in various crystal matrices.

Experiments with LiF, described in Progress Report No. XXII, were repeated with the more sensitive equipment. The previous results were confirmed (Fig. 12). There is no change of sign in the derivative of the dis persion resonance. The crystal is very strongly saturated and no curve resembling resonance absorption was obtained. Dispersive contributions arising frơr bridge drifts or slight errors in balancing the bridge predominate.

$\bullet$.

3. Ferromagnetic resonance in magnetite (P. A. Miles)

Double resonance-loss peaks occur in magnetite below the $118^{\circ} \mathrm{K}$ transition. The dependence of this structure in resonance dispersion $\chi^{\prime}$ on crystal orientation has been observed at $90^{\circ} \mathrm{K}$ and is being analyzed. 
4. Resonances in garnet and ruby at $10 \mathrm{kMc}$ (B. E. DePalma and T. E. McEnally)

A spectrometer using 6-kc magnetic-field modulation has been set up to operate at $10 \mathrm{kMc}$ (Fig. 13). A tunable reflection cavity is used, operating in the cylindrical TE 011 high $-Q$ mode. Originally the field modulation was

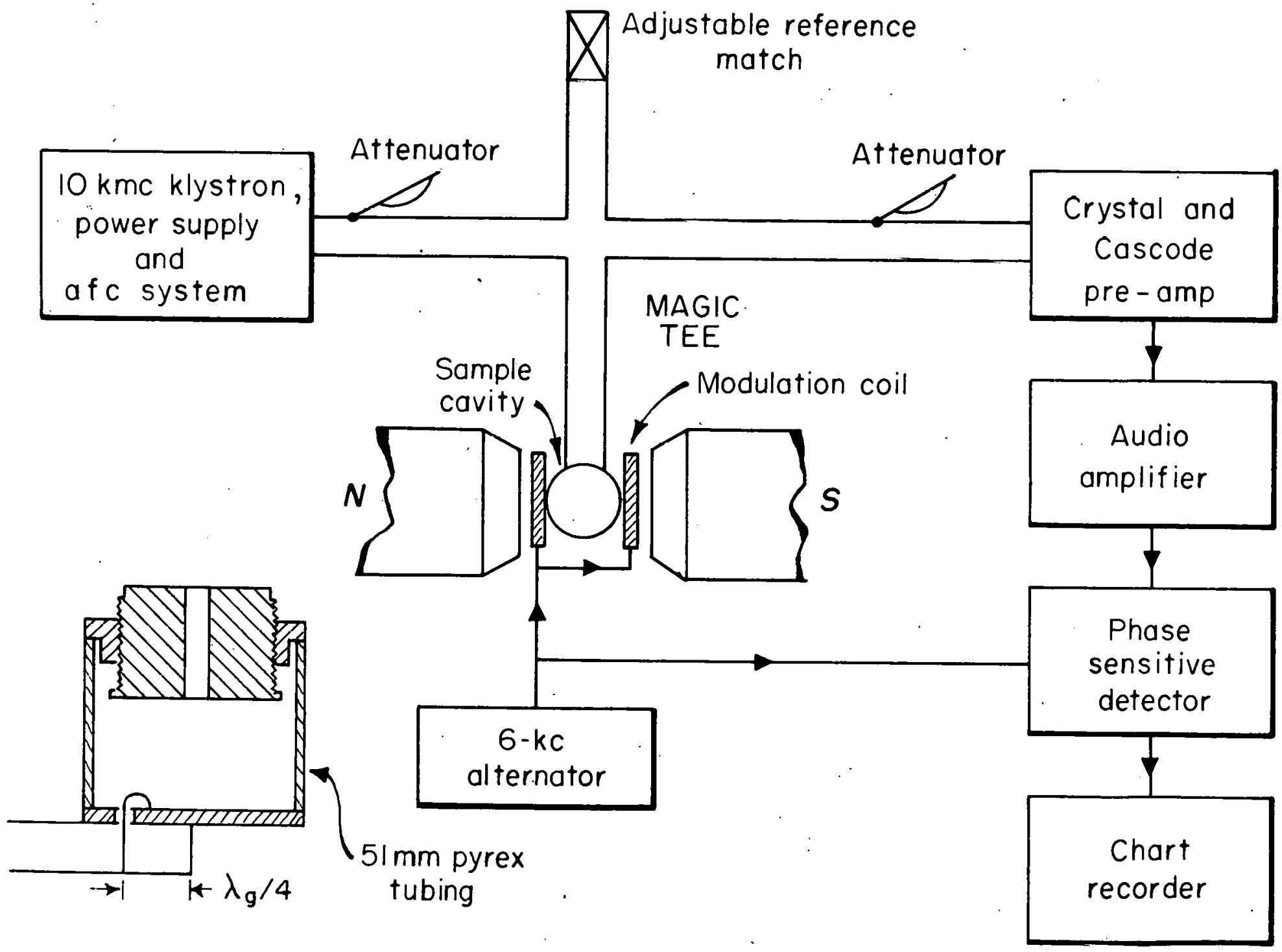

(b)

(a)

Fig. 13. Magnetic spectrometer for $10 \mathrm{kMc}$. (a) Block diagram of equipment. (b) Sectional view of cavity for containing short rod samples. The cavity is magnetically coupled to waveguide with a loop. 
produced by coils mounted within the cavity itself, but this led to spurious modulation signals due to movements of the coil and to a significant lowering of the cavity $Q$. A gold-plated cavity with glass walls has now been substituted, thus permitting the modulation field from exterior coils to penetrate the cavity walls. Samples may be mounted through holes in the cavity end plates, an arrangement which allows insertion of glass Dewars for measurements at low and high temperatures.

The limiting noise source, as in the higher frequency spectrometer, is the incident klystron power. This is probably true of all such high-' sensitivity systems, a fact usually ignored in discussions of the merits of various spectrometer techniques.

A pulsed magnetron source in the form of a modified radar set has been set up to provide high-power microwave pulses for the study of relaxation effects in ferrimagnetics. We are at present looking for magnetization changes produced by these pulses in spheres ground from yttrium-iron garnet crystals.

As part of the program on the behavior of paramagnetic ions in oxide lattices, confirmatory spectra from $\mathrm{Cr}^{3+}$ ions in corundum (synthetic ruby). are being obtained.

\section{HIGH-FIELD-STRENGTH RESEARCH}

1. Conduction in hexane (M. Bono)

Further purification of hexane was undertaken in extending our studies on the effect of impurities on current-voltage characteristics (cf. Progress Report No. XXII).

Various methods were tried to eliminate benzene from the hexane:

(a) formation of an adduct between hexane and urea, with subsequent distillation 
and collection of the hexane contained in the adduct; (b) slow crystallization. of the hexane in liquid nitrogen; (c) percolation of the hexane through a column of silica gel or alumina and filtration through an ultrafine frittedglass filter.

Only the last method gave a product which no longer showed the three absorption peaks in the ultraviolet which are characteristic of benzene.

Unfortunately, the current-voltage characteristic obtained with the hexane thus purified does not appear to differ significantly from characteristics obtained with the impure hexane. We are concentrating now on electrical purification methods.

2. Photoconductivity and space-charge build-up in thallium bromo-iodide $($ KRS-5) (R. M. Moon and D. A. Powers)

A study of the response of photocurrent in KRS-5, similar to that previously reported for the alkali halides, ${ }^{2,3)}$ has been initiated. Preliminary measurements at room temperature, using the apparatus of von Hippel et al. 2) show current transients which appear to result from two competing effects: a rising transient with a time constant increasing with light intensity and decreasing with field strength, and a falling transient with a time constant decreasing with light intensity and field strength. These preliminary observations were made with unfiltered light from a tungsten projection lamp. To separate the two effects, monochromatic light will be used.

Since at room temperature the dark current is an appreciable fraction of the total current, a new test cell has been designed and constructed with guarded electrodes to eliminate surface leakage. Furthermore, the measurements are being extended to liquid-nitrogen temperature.

2) A. R. von Hippel, E. P. Gross, J. G. Jelatis, and M. Geller, Phys. Rev. 91, 568 (1953).

3) M. Geller, Phys. Rev. 101,1685 (1956).

$$
50 \% \therefore 8
$$


At $300 \mathrm{v} / \mathrm{cm}$, after a rapid initial rise, the current became very noisy and continued a slow, irregular rise reaching $2 \frac{1}{2}$ times its initial value in 6 min. with no indication of approaching a steady state. This behavior, similar to that reported by Hofstadter, will be studied in the new test cell to decide whether it is a surface or a bulk phenomenon.

3. Preparation of thin films of fused quartz and Vycor glass ( $\mathrm{J}$. Kalnajs)

Thin flat films of Resistor and ordinary Pyrex glass, made by heating and stretching, were used in our preliminary study on the dielectric strength of glass layers. These materials are multicomponent systems. Sodium, present in varying concentrations, is one of the undesirable constituents, causing high ionic conductivity and poor surface conditions.

During the heating the sodium, by evaporation, becomes more depleted in the surface layers. Thus the thinner sections of the film contain less sodium than the thicker, and the film becomes inhomogeneous as. regards the sodium. Fused silica and Vycor glass would be preferred but their high softening temperature and the narrow working interval make them difficult to handle. Trapped microscopic bubbles, especially in Vycor glass, are another unpleasant feature, because they form weak spots and lead to puncture of the film. On the basis of the experience gained, we succeeded in producing Vycor and quartz films as thin as 1000A. Commercial tubing (Corning 7900 glass and Amersil fused silica, 7 to $9 \mathrm{~mm}$ o.d.) served as starting material. The bubble technique (cf. Progress Report No. XIX) was used in the two-dimensional stretching operation. The material, heated to the working point by two oxyhydrogen flames, was blown very fast to the desired thickness. While the silica or Vycor diaphragms have a clear appearance when made, opening the envelope with a needle flame for measure4) R. Hofstadter, Phys. Rev. 72, 1120 (1947). 
ment of the film thickness produces an undesirable deposit of silicon monoxide-dioxide on them. New techniques to avoid this difficulty are under examination.

4. Electric conductivity in glass films (C.W. Nelson)

Films, 0.2 to $0.4 \mu$ thick, of Corning 1728 aluminosilicate glass were provided with a gold anode and silver iodide cathode. The silver iodide cathode was formed by exposing a thin film of silver on glass lu iodine vapnr. This cathode was found to be free from field emission, as expected, since AgI is an ionic conductor. Current-time measurements at roum temperature, with voltage as a parameter, showed greatly reduced or no noise compared with metallic gold cathodes and the final current was reduced. Charge current vs. time characteristics (Fig. 14) at various voltages for a silver iodide cathode on aluminosilicate glass ca. $0.2 \mu$ thick showed this reduction (cf. Progress Report No. XXII, Fig. 13, for results obtained with gold electrodes).

Some of the Corning Vycor glass provided with gold electrodes proved to be also free from noise.

5. Dielectric breakdown of alkali halides (L. R. Schissler)

The coaxial sample holder described in Progress Report No. XXII, p. 22, has now been completed and is under test. Preliminary measurements on $\mathrm{KBr}$ have confirmed the earlier finding that exposure of $\mathrm{KBr}$ to water vapor increases the breakdown field strength. KBr samples stored in a rroist atmosphere gave values for the breakdown field of $0.83 \mathrm{Mv} / \mathrm{cm}$ for slow pulses in agreement with 0.79 and 0.88 for various "wet" conditions reported by von Hippel and Alger in Technical Report 16.

For the measurement of prebreakdown currents, a sample holder was connected to a stabilized d-c power supply and a protecting circuit, which 


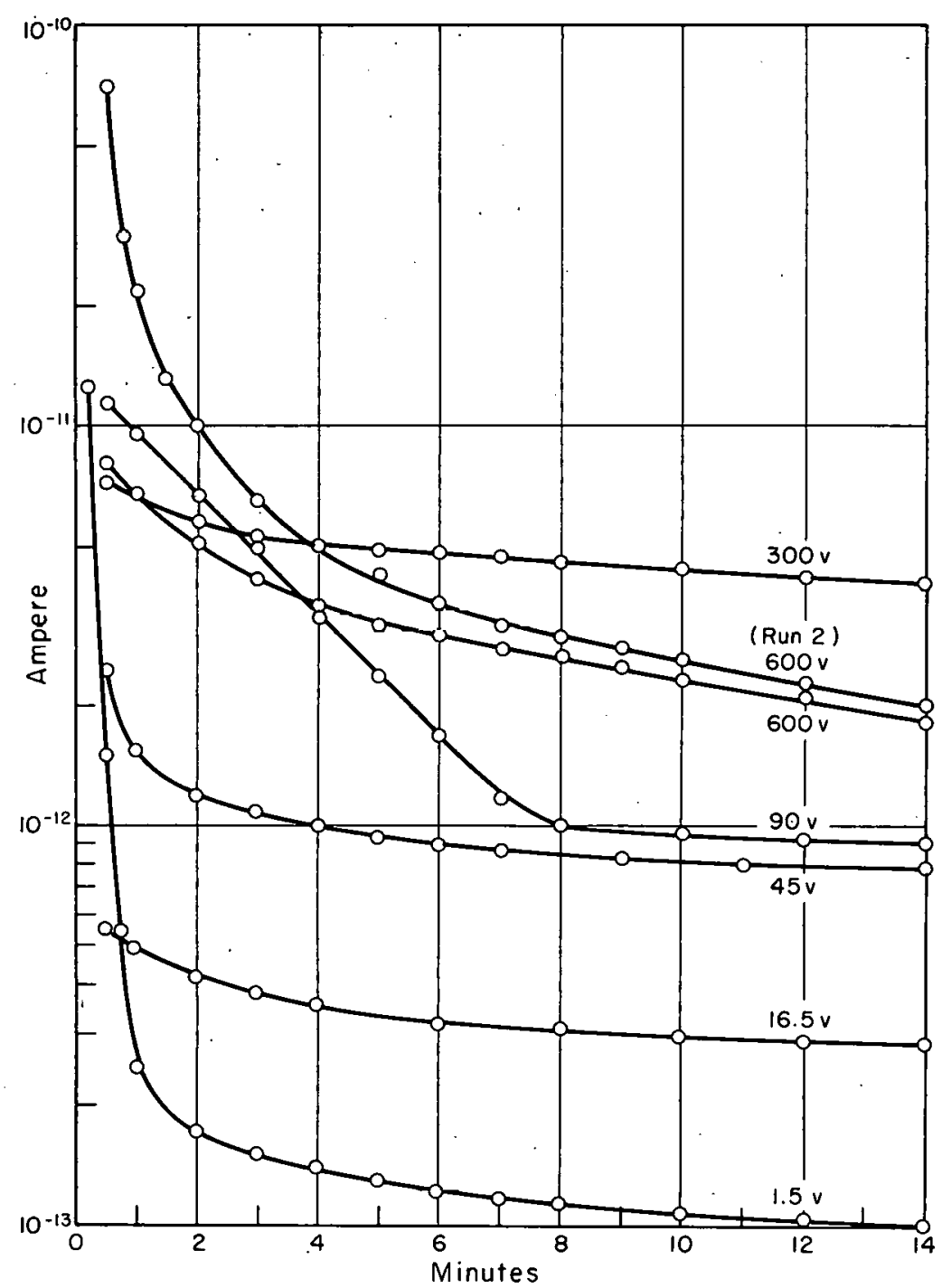

Fig. 14. Charge current vs. time characteristics for various voltages for silver iodide cathode on Corning 1728 aluminosilicate glass.

removes the high voltage quickly after breakdown.

6. Interaction of electrons and holes with lattice ions in polar crystals (S. J. Nettel)

A promising approach to the theory of electric breakdown in polar crystals is the study of the behavior of slow charges. The present aim is to find the nature of quantum-mechanical states of a charge-lattice system, in 
which the polar lattice is deformed about an excess or defect charge. For convenience we choose as the charge a. $3 \mathrm{p}$ hole in the valence band of a $\mathrm{KCl}$ crystal. Our analysis may also have a bearing on the discussion of the spin-resonance absorption spectrum found by Kunzig. ${ }^{5)}$

A crystal wave function that contains the desired correlation between the motion of the hole and of mobile crystal ions has been found and the formal work of determining the expectation value of the total energy of the crystal has been largely completed. This energy is being minimized with respect to those parameters in the wave function that give the extent of the lattice deformation. Numerical calculations will soon be started. The work is proceeding under the guidance of Professor G. F. Koster of the Solid State and Molecular Theory Group.

7. Field strength effects in cadmium sulfide single crystals (H. Gildea) Previous experiments on cadmium sulfide have indicated that the conduction and breakdown processes may involve field distortion due to space charge, impact ionization, field emission, and electronic recombination levels. The application of electric fields across single crystals of cadmium sulfide without optical excitation gives important clues to understanding these mechanisms in an ionic semiconductor.

In this research, an extensive series of current-voltage-time and related measurements at various temperatures was made on cadmium sulfide single crystals. A simplified approach, using d-c and unidirectional pulse fields, was adopted. For most measurements, the crystals were not optically excited, but controlled illumination and thermal excitation were sometimes employed to study transient effects.

Crystals of two general types were found. Most crystals had a rather

5) W. Kunzig, Phys. Rev. 99, 1890 (1955). 
high conductivity, essentially independent of temperature in the range $-175^{\circ}$ to $100^{\circ} \mathrm{C}$ (Fig. 15). However, one crystal had a low conductivity which was exponentially dependent on temperature (Fig. 16). D-c and unidirectional

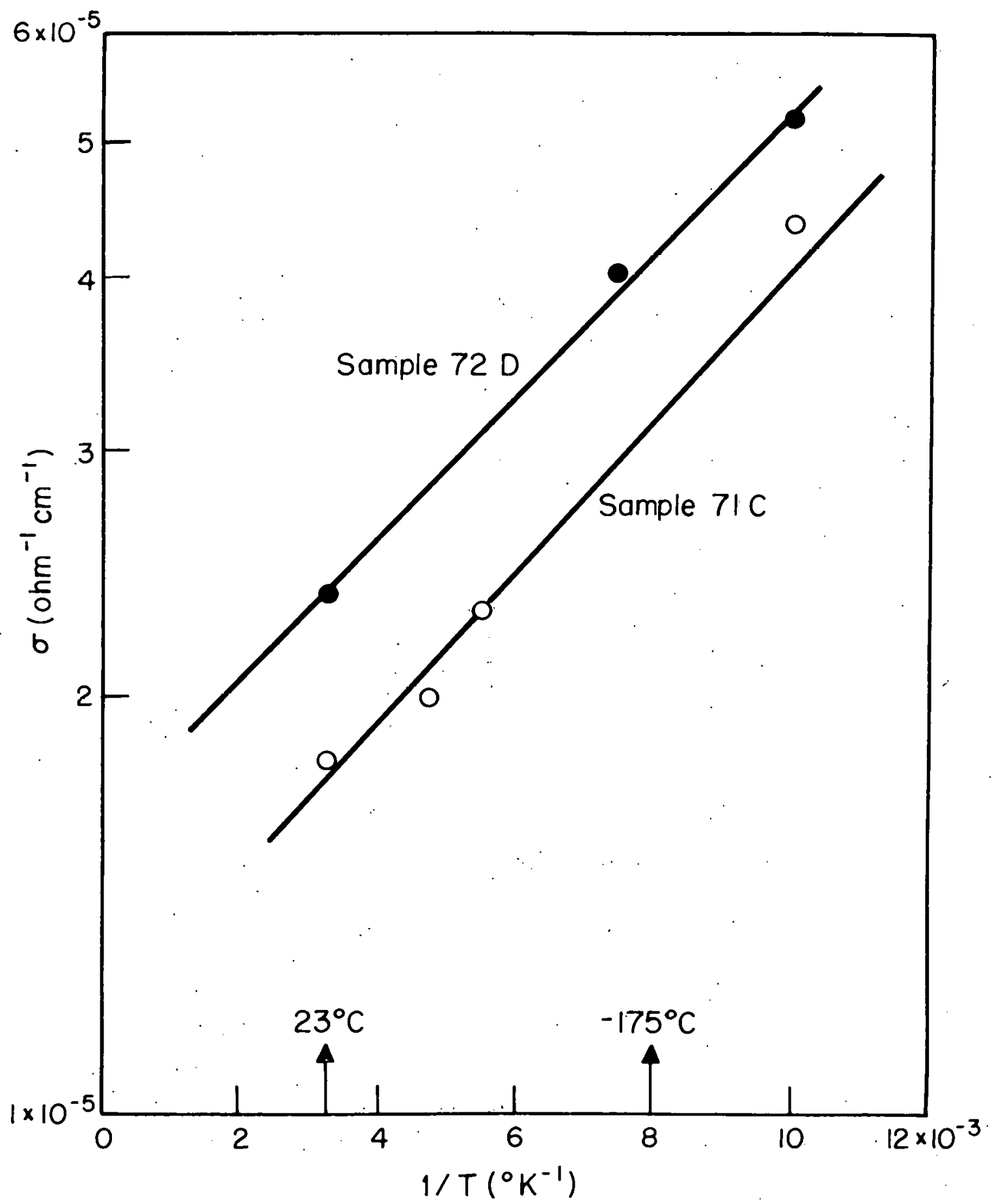

Fig. 15. Dark conductivity vs. temperature, samples $71 \mathrm{C}$ and $72 \mathrm{D}$. 
pulse current-voltage characteristics of the high-conductivity crystals exhibited a linear form up to a certain point where a negative dynamic resistance region vas observed (Fig. 17). Repeated excitation would generally move the breakover point to a lower current or lower voltage. The original conditions

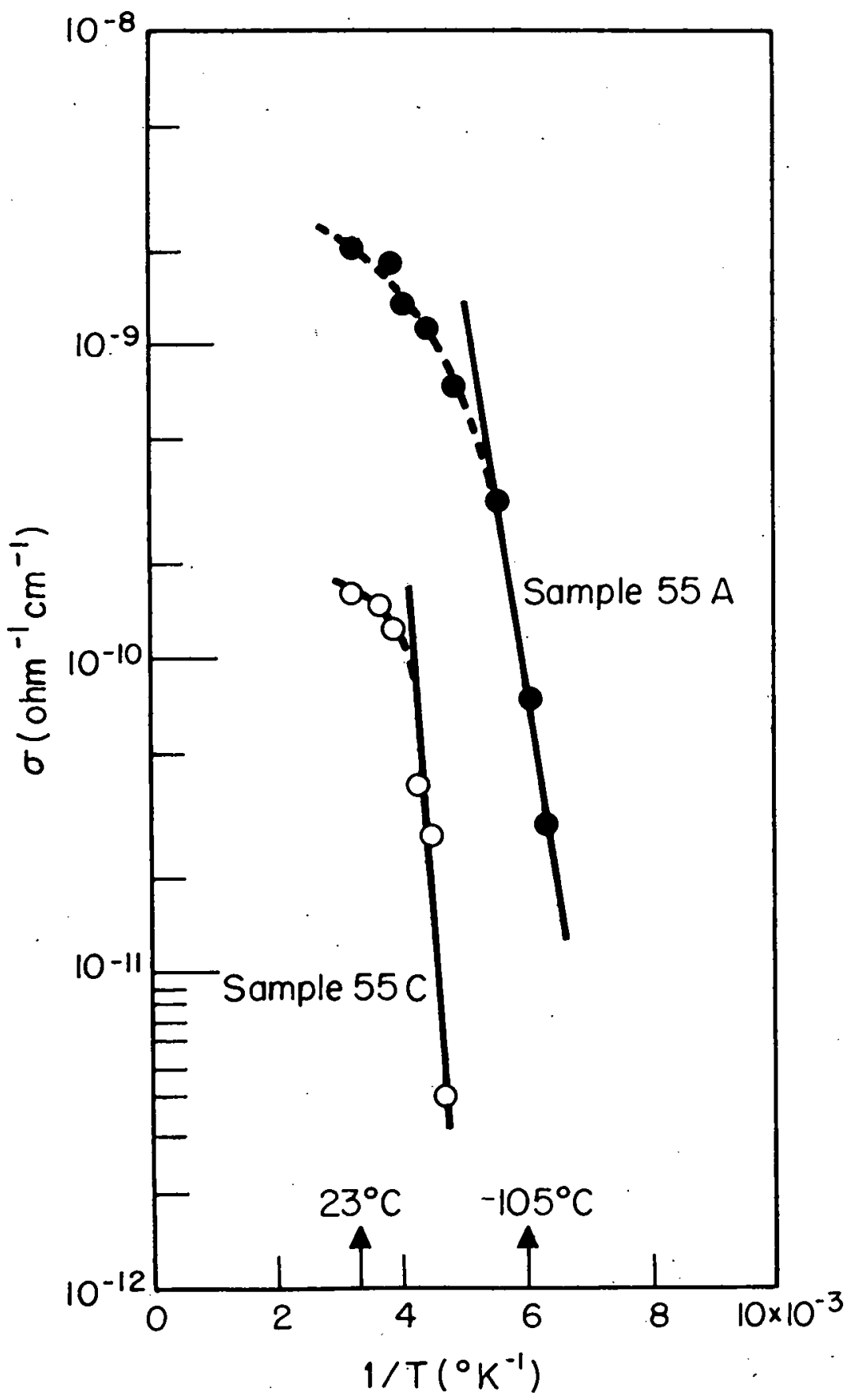

Fig. 16. Dárk conductivity vs. temperature, samples $55 \mathrm{~A}$ and $55 \mathrm{C}$. 


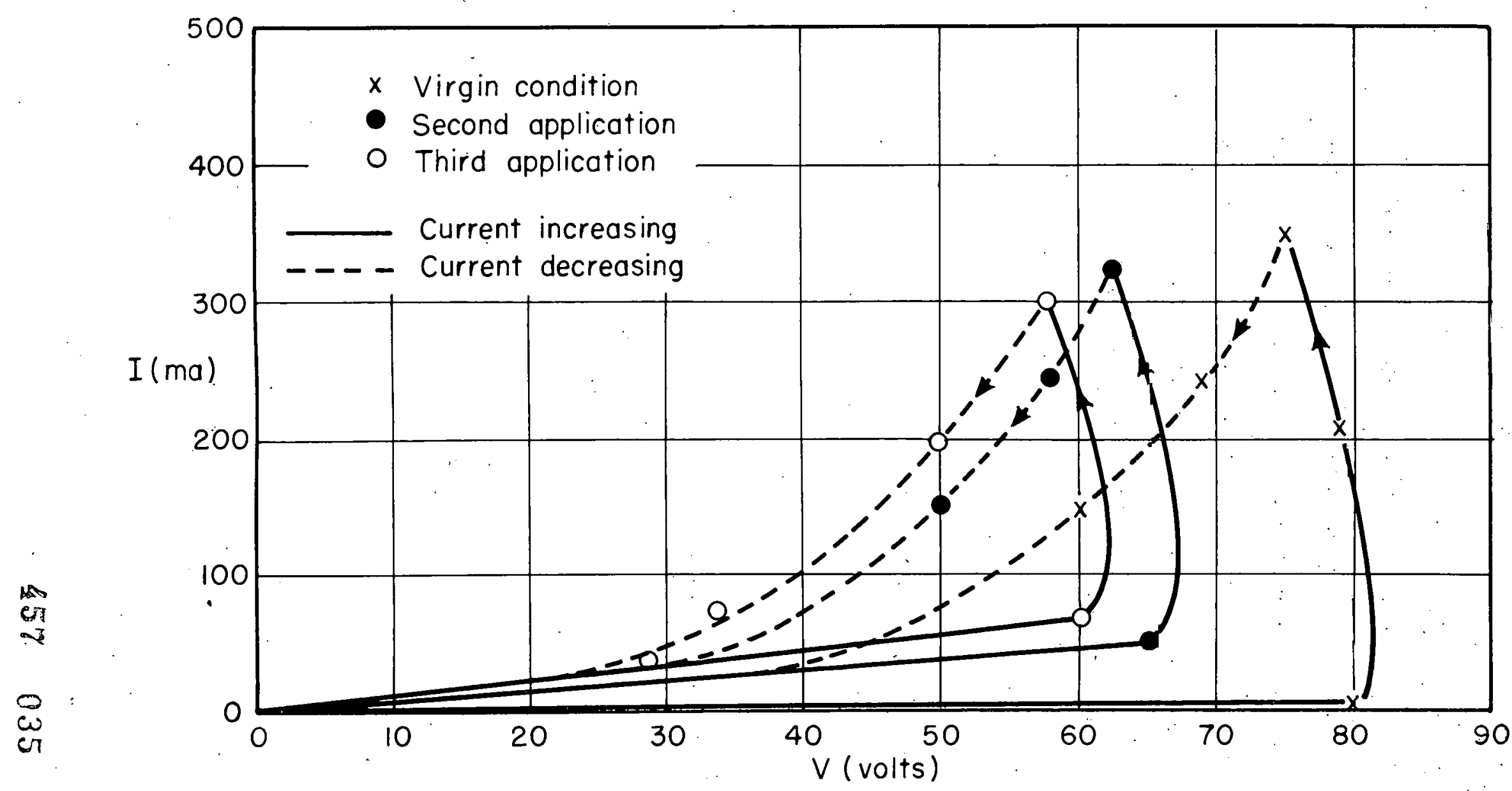

Fig. 17. Hysteresis and variation of breakdown field in sample $72 \mathrm{~B}$. 
could be restored by bulk heating or by letting the crystal remain for rather long periods without electrical excitation.

The current-voltage characteristics of the low-conductivity crystals were generally in qualitative agreement with those of the high-conductivity crystals. The negative dynamic resistance region and semipermanent, but reversible, changes in electrical characteristics were observed (Fig. 18). However, when a step voltage was applied to a low-conductivity sample, a decaying current transient, associated with space-charge polarization, was observed. Photo-excitation experiments indicate that positive space charge can be neutralized by photo-electrons, restoring the virgin condition in relatively short periods of time.

Many of the results are in agreement either with those found by Smith and Rose or those found by Diemer, but not with both. The effect of thermal and optical excitation in restoring the virgin characteristics of a cadmium. sulfide crystal has not been previously reported.

\section{FERROELECTRICS AND FERROMAGNETICS}

\section{A. Ferroelectrics}

On the reversal of the spontaneous polarization in barium titanate (P. Pearle)

In addition to the work reported under Dielectric Spectroscopy, a master's thesis on the switching properties of $\mathrm{BaTiO}_{3}$ was carried through as a VI A thesis at the Bell Telephone Laboratories in continuation of the work of E. A. Little (Technical Report 87, Oct., 1954).

A train of square voltage pulses, each reversing only a small fraction of the total reversible spontaneous polarization (S.P.), was applied to a c-domain crystal and the amount of S.P. reversed (during a pulse on-time and off-time) was measured. It was found that under certain conditions, S.P. 


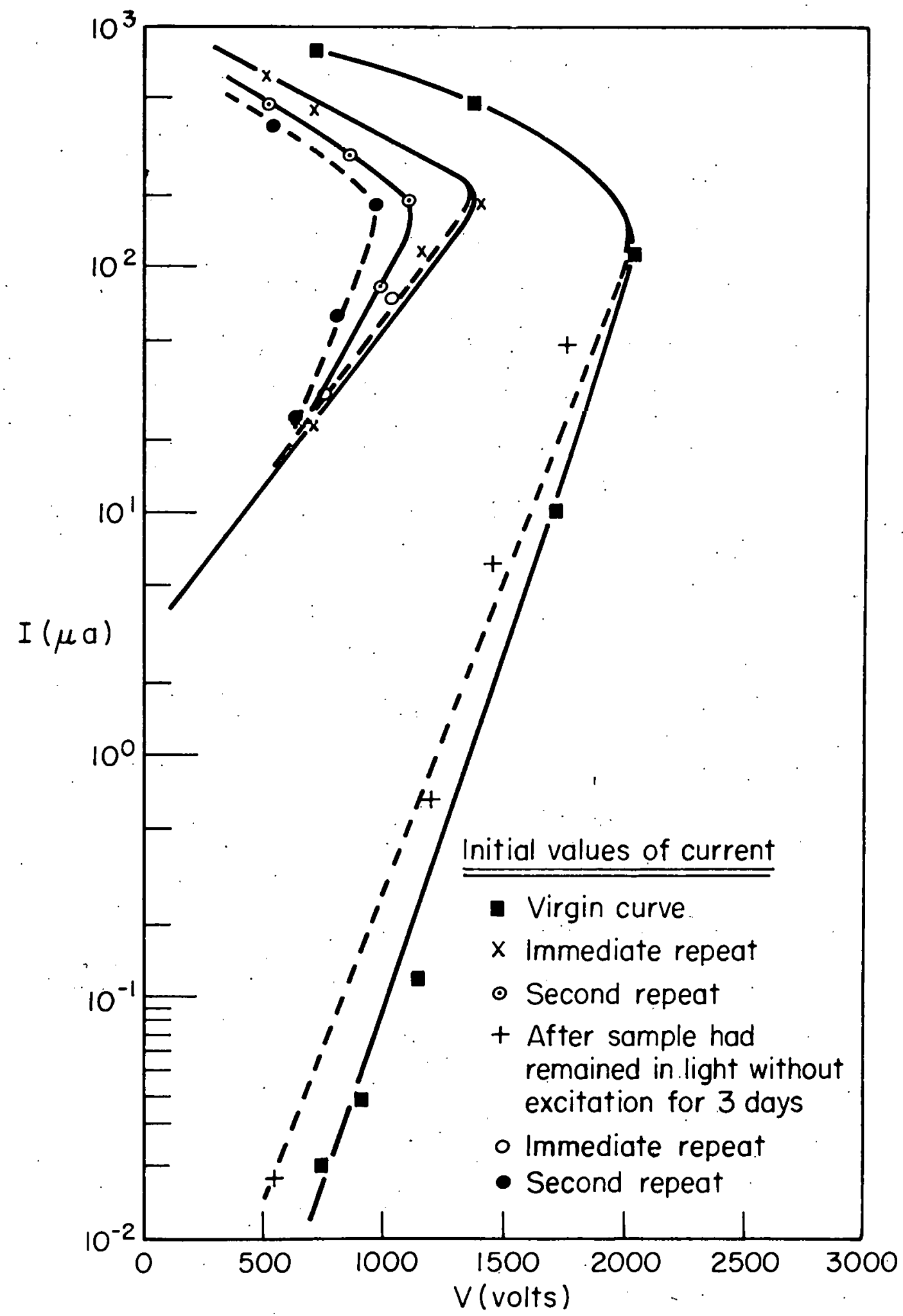

Fig. 18. D-c characteristics of sample 5,5D at room temperature. 
which had been reversed by an applied pulse would, when the pulse was removed, reverse "by itself" back to its original state. On a domain model, this "backswitching" implies a process whereby domain walls move without an externally applied field.

Experiments were conducted on two classes of crystals: ca. 2 mils thick with $21 \times 21 \mathrm{mil}$ square coin-silver electrodes, and 4.5 to 10 mils thick with ca. 100 mil diameter LiCl electrodes. Backswitching occurred in the metal electroded crystals and not in the liquid electroded crystals. It was found that backswitching could be characterized by a parameter $G \leqslant 1$, the ratio of net S.P. reversed by the pulse during the on-off cycle to the net S. P. reversed by the pulse during the on-time. Backswitching increased (G. decreased) with increasing temperature and decreased with increasing pulse length and pulse height. A d-c field (as small as $20 \mathrm{v} / \mathrm{cm}$ ) aiding the pulse field (ca. $600 \mathrm{v} / \mathrm{cm}$ ) decreased backswitching considerably.

Two qualitative models which might account for backswitching are discussed. One invokes the local fields caused by free charge carriers as a mover of domains. The other considers the domains as moving to equilibrium positions where the potential energy of the crystal has a stationary value; the energy is taken as the sum of strain and electrostatic energies.

\section{B. Ferromagnetics}

1. Magnetic properties of yttrium-iron garnet (B. Frackiewicz and D. J. Epstein)

Magnetic relaxation spectra similar to those occurring in certain nickel-zinc ${ }^{6)}$ and magnesium (Progress Report No. XVII, June, 1955, p. 23) ferrites have been observed in polycrystalline yttrium-iron garnet ${ }^{7)}$ (YIG).

6) D. J. Epstein, Conference on Magnetism and Magnetic Materials, AIEE Publication T-91, Feb., 1957, p. 493.

7) The samples of YIG were obtained from the Bell Telephone Laboratories through the co-operation of $\mathrm{Dr}$. M. A. Gilleo. 


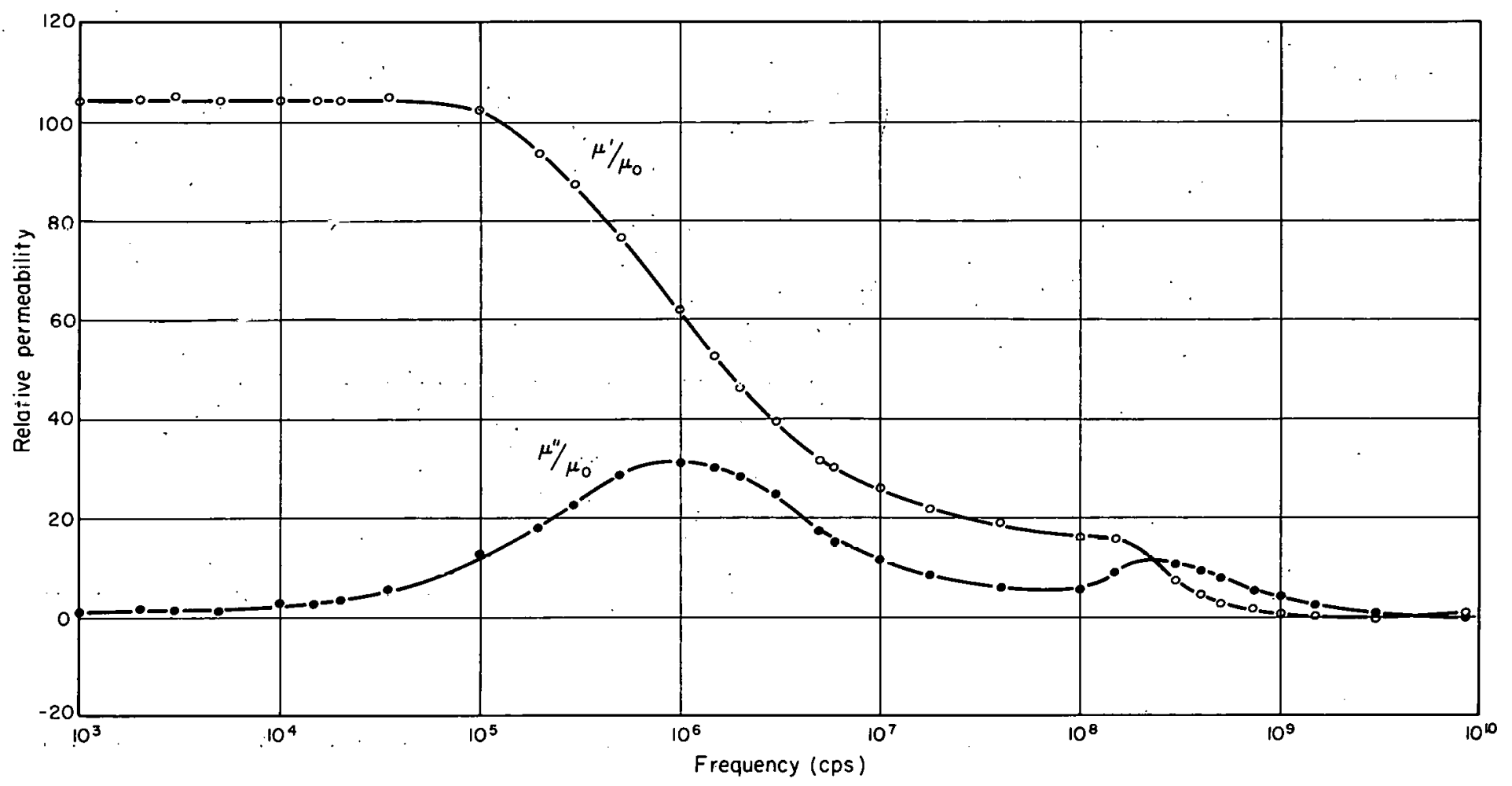

Fig. 19. Initial permeability at room temperature of polycrystalline yttrium-iron garnet $\left(3 \mathrm{Y}_{2} \mathrm{O}_{3} \cdot 5 \mathrm{Fe}_{2} \mathrm{O}_{3}\right)$.

Room-temperature measurements of complex initial permeability, obtained in this laboratory by Westphal and East (Fig. 19), show two well-defined regions of dispersion. The principal one, occurring in the radio-frequency region, we associate with a domain wall relaxation process; the microwave dispersion is probably of gyromagnetic origin.

Our main interest, at the moment, is centered on the radio-frequency branch of the spectrum. When the results of Fig. 19 are replotted as a ColeCole diagram (Fig. 20), the low-frequency dispersion presents a circular arc with its center below the horizontal axis. For such a locus the complex permeability can be represented functionally by the relaxation formula: ${ }^{8}$

$$
\mu_{\infty}^{*}=\mu^{\prime}+\left(\mu_{s}^{\prime}-\mu_{\infty}^{\prime}\right) \int_{0}^{\infty} \frac{g(z)}{1+j \omega \tau}\left(\frac{d \tau}{\tau}\right),
$$

8) K. S. Cole and R. H. Cole, J. Chem. Phys. 9, 341 (1941).. 


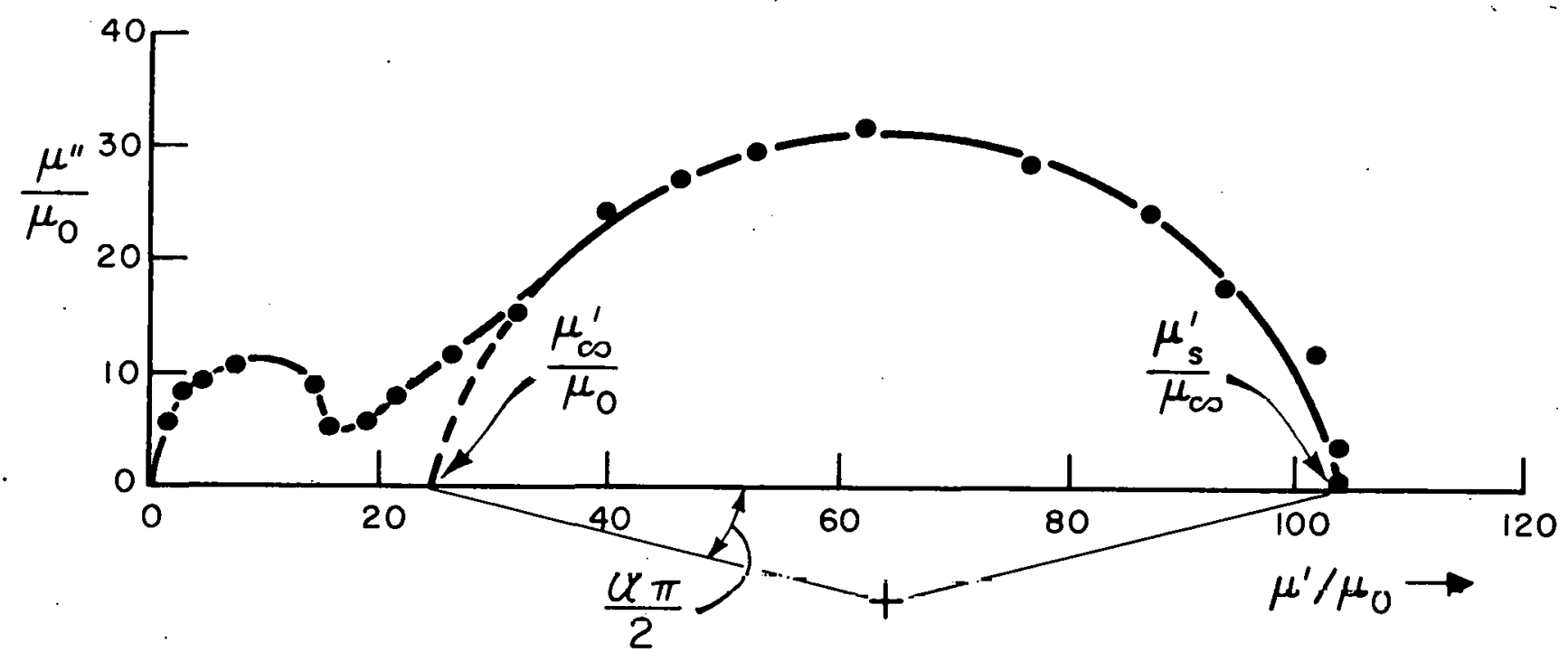

Fig. 20. Cole-Cole diagram for permeability of yttrium-iron garnet.

where $\mu_{s}^{\prime}$ and $\mu_{\text {cs }}^{\prime}$ are respectively the permeabilities in the low - and highfrequency limits (Fig. 20) and where $g(z)$ represents a distribution of time constants given by

$$
g(z)=\frac{1}{2 \pi} \frac{\sin a \pi}{\cosh (1-a) z-\cos a \pi} .
$$

In this expression, $a \pi$ is defined in Fig. 20 and $z=\ln \left(\tau / \tau_{0}\right), \tau_{0}$ being the central time constant in the distribution. It is clear from Eq. (2) that the distribution function is logarithmically symmetrical in $\tau$. For our specimen of YIG the distribution, as measured between $1 /$ e points, contains a $5: 1$ range of-time constants. This spread in $\tau$ is a factor 20 to 30 times smaller than. we have observed previously in polycrystalline samples of nickel-zinc ferrite. As in the nickel-zinc and magnesium ferrites, the permeability spectrum of the garnet is strongly temperature dependent. The effect of temper ature was studied by making a series of permeability runs as a function of temperature at several different fixed frequencies. ${ }^{*}$ A typical result is

\footnotetext{
* These measurements were made by E. R. Steinhart as part of his S. B.
} thesis in Electrical Engineering, Mass. Inst. Tech., May, 1958. 


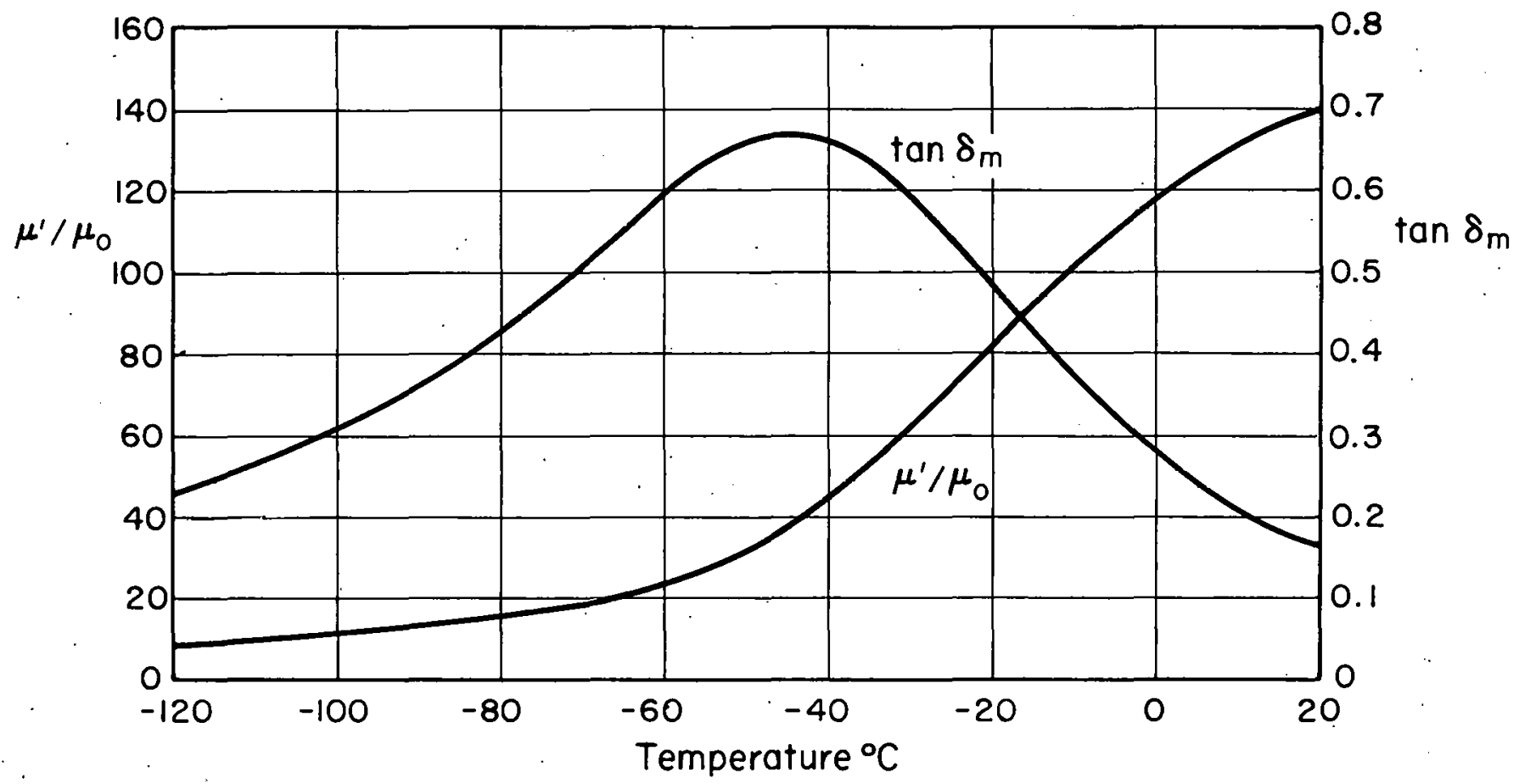

Fig. 21. Initial permeability and loss tangent of yttrium-iron garnet as a function of temperature, measured at $0.1 \mathrm{Mc}$.

shown in Fig. 21. The temperature corresponding to the peak in the magnetic loss tangent $\left(\tan \delta_{m}\right)$ shifts with frequency according to a Boltzmann relation

$$
\nu=c e^{-U / k T} \text {. }
$$

The activation energy $U$ for the process, according to Fig. 22, is $0.25 \mathrm{ev}$. In the nickel-zinc ferrites the activation energy for magnetic relaxation was equal to that for electronic conduction. This will be checked for YIG. At $20^{\circ} \mathrm{C}$ the sample resistivity is $3 \times 10^{6} \mathrm{ohm}-\mathrm{cm}$ (measured at $100 \mathrm{cps}$ ), an indication that the sample is probably slightly reduced.

At liquid nitrogen temperature an extremely slow switching behavior is observed, The change in magnetization for a step reversal of the magnetizing force from $-\mathrm{H}_{\mathrm{m}}$ to $+\mathrm{H}_{\mathrm{m}}$ is shown as function of time in Fig. 23. 


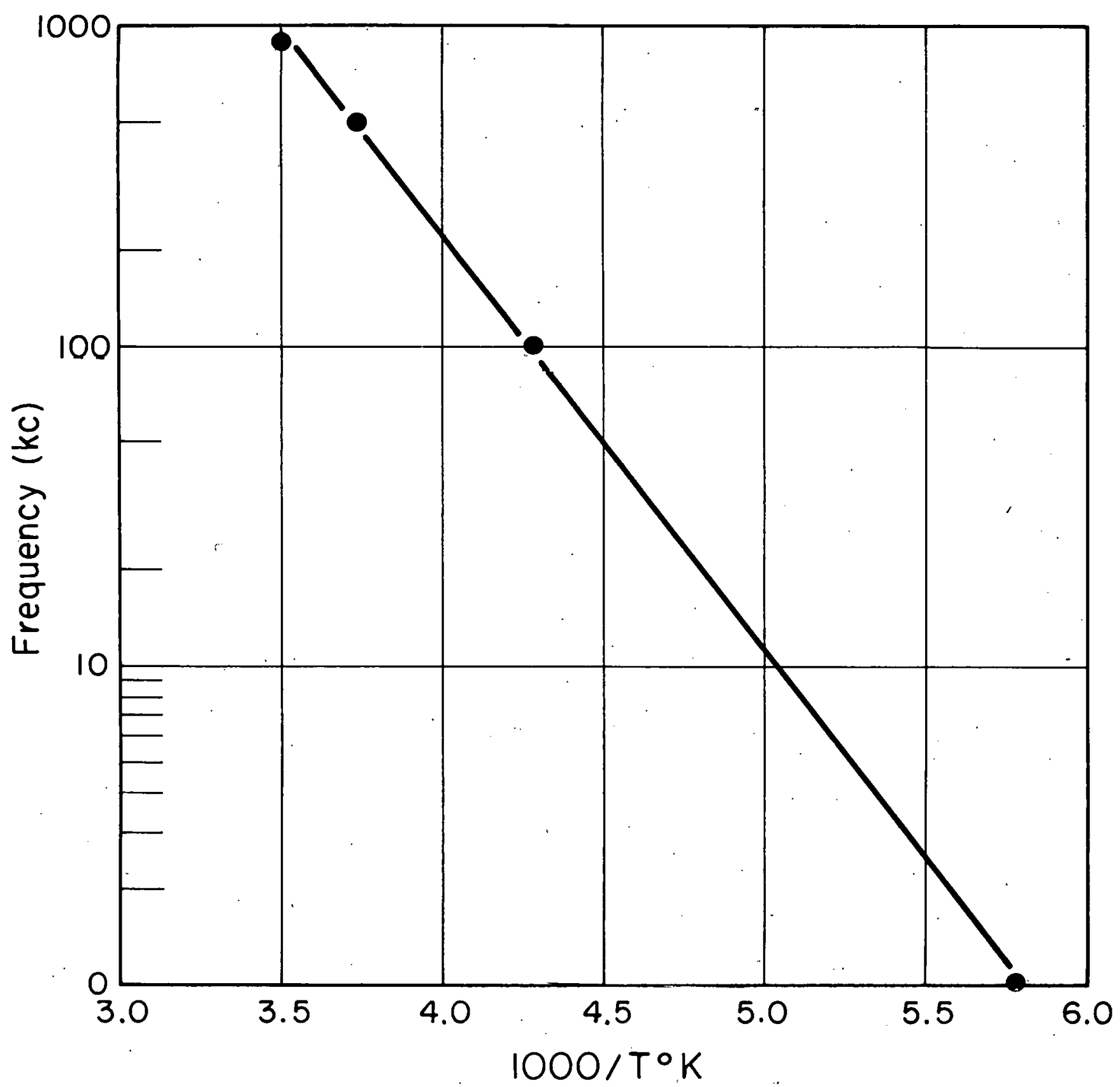

Fig. 22. Shift in maximum of loss tangent with frequency in yttrium-iron garnet.

Considerable difficulty has been experienced in obtaining closely reproducible permeability and switching results. The material appears to be very sensitive to its thermal and magnetic prehistory. 


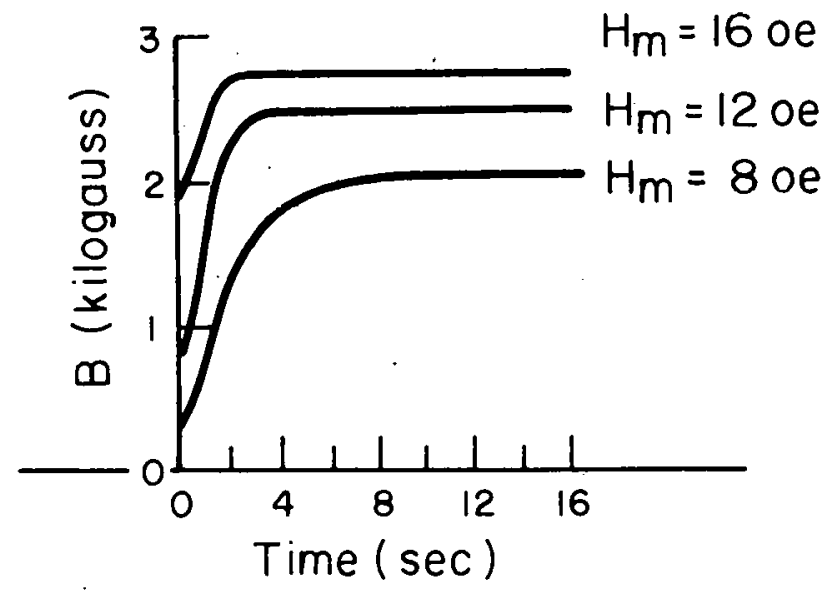

Fig. 23.

Change of magnetization with time for a step reversal of $\mathrm{H}$ from $-\mathrm{H}_{\mathrm{m}}$ to $+\mathrm{H}_{\mathrm{m}}$ for polycrys talline yttrium-iron garnet.

2. Magnetic oxides under high pressure (T. P. Janusz and P. W. Forsbergh, Jr.)

The intensifier section of the pressure apparatus has been completed. and is ready for test. The differential transformer has been tested. The detecting circuit is shown in Fig. 24 and the micrometer adjustment for the differential transformer in Fig. 25. With this system it is possible to detect a displacement of $2 \times 10^{-5}$ inch.

A temperature bath employing a stainless steel Dewar, similar to a

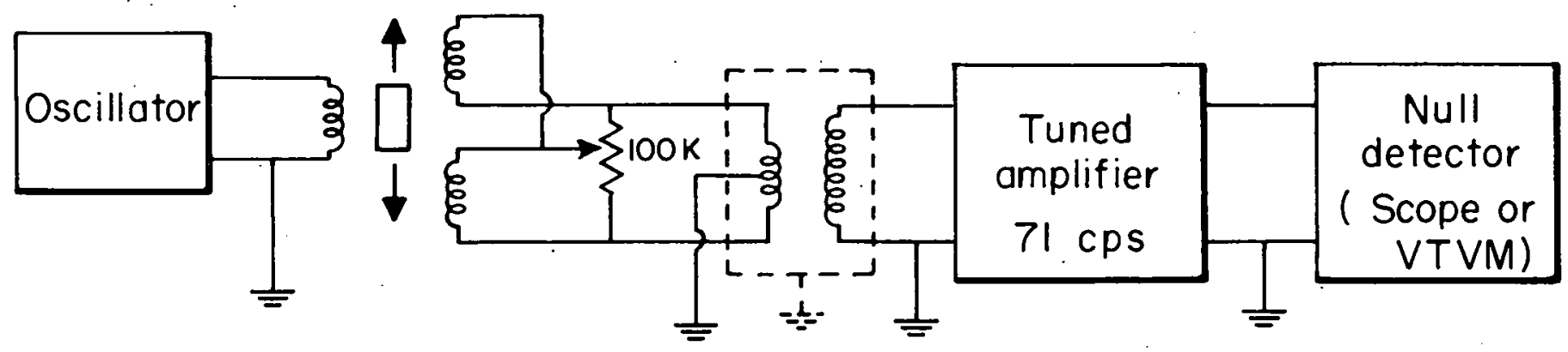

Fig. 24. Differential transformer null-detection circuit used in conjunction with the micrometer adjustment. 


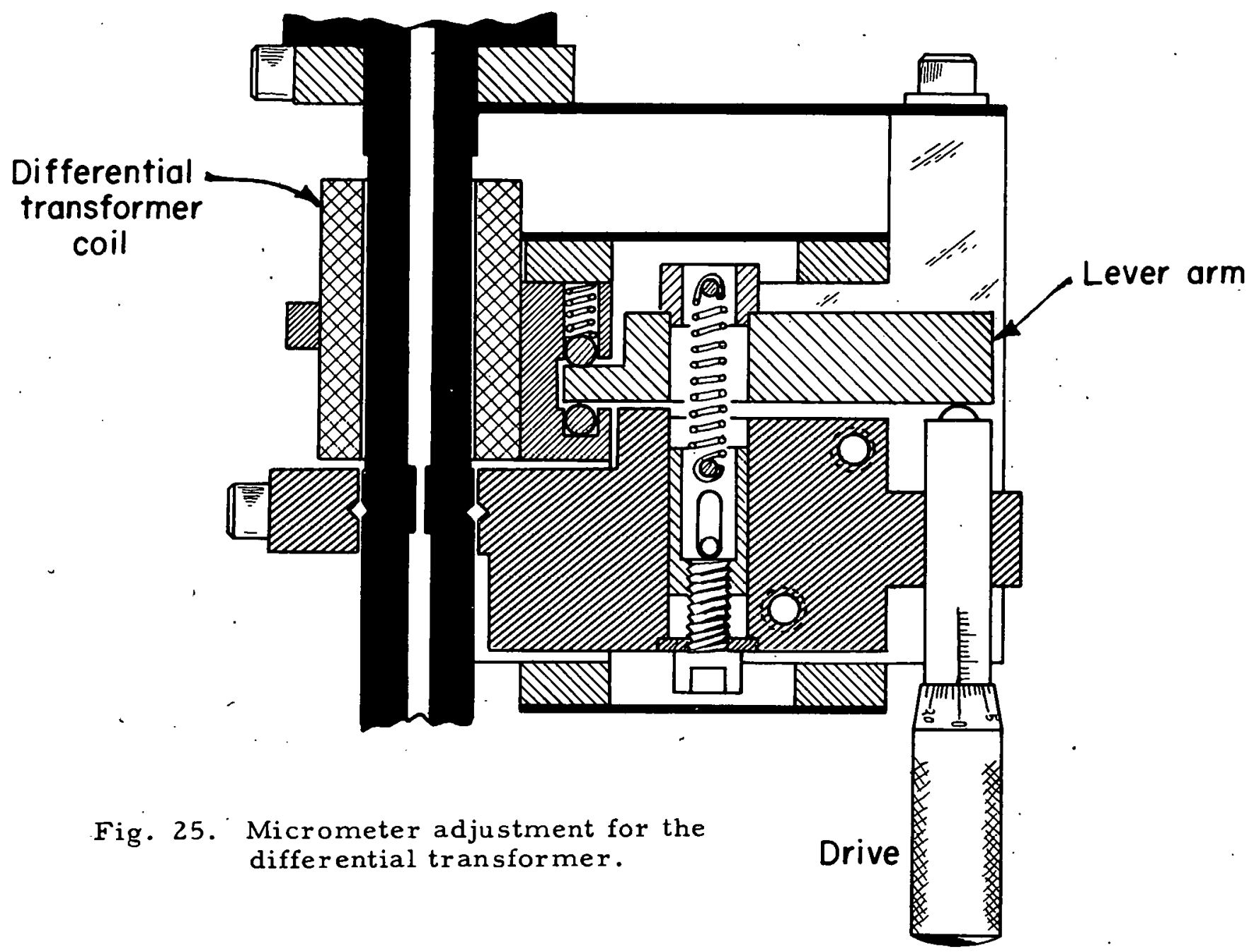

design by Fuschillo and Krautkopf, ${ }^{9)}$ is under construction; the expected range i's $-180^{\circ}$ to $350^{\circ} \mathrm{C}$.

Preparation of transition metal oxides has started. Ferrous oxide is made by the thermal decomposition of iron oxalate according to $\mathrm{FeC}_{2} \mathrm{O}_{4} \stackrel{\Delta}{\longrightarrow}$ $\mathrm{FeO}+\mathrm{CO}+\mathrm{CO}_{2} \cdot$ The $\mathrm{CO}$ and $\mathrm{CO}_{2}$ are carried off in a stream of nitrogen in order to prevent reduction of the $\mathrm{FeO}$ by the CO.

9) N. Fuschillo and D. W. Krautkopf, Rev. Sci. Instr. 28, 1060 (1957). 


\section{SEMICONDUCTORS}

1. Optical properties of the isoelectronic compounds of germanium ( $R$. Coelho)

The main purpose of the research is to investigate, by optical methods, the relation between polarity of lattice and band structure; in particular, we wish to study the effect of polarity on the exciton states. For this purpose comparative work has been started on homologues of the germanium series, where the ionic character increases from $\mathrm{Ge}$ to $\mathrm{CuBr}$ while the crystal structure and about the same lattice constant are maintained.

For germanium itself we can rely on published data. According to the most recent observations, both the indirect and the direct optical transition show an excitonic structure. For gallium arsenide the conduction band minimum is at the center of the Brillouin zone and the situation may be appreciably simpler. Since investigations of this compound are in progress at several other laboratories, we are concentrating at present on zinc selenide ( $\mathrm{ZnSe}$ ) and cuprous bromide ( $\mathrm{CuBr}$ ). Little is known about $\mathrm{ZnSe}$; its close neighbor $\mathrm{ZnS}$ has been studied extensively and $\mathrm{ZnTe}$ has been investigated recently. The optical absorption edges of both $\mathrm{ZnS}$ and $\mathrm{ZnTe}$ show some structure. Thin films of cuprous bromide show strong absorption bands, ${ }^{10}$ ) probably connected with exciton transition.

It has been shown that in a covalent lattice the exciton can be considered as a hydrogen-like two-body system of mass equal to the reduced effective mass of the electron and hole and with a volume extending over several unit. cells. An exciton in an ionic crystal is localized on one unit cell, the excited electron being bound to one of the positive ion neighbors of the negative ion where the hole stays trapped. Overhauser, ${ }^{11)}$ using group-theoretical

10) S. Nikitine et al., Compt. rend. 242, 1003 (1956); 242, 1588 (1956); 245, $52(19.57)$.

11). A. W. Overhauser, Phys. Rev. 101, 1702 (1956). 
arguments, has classified the possible states of the exciton in $\mathrm{NaCl}$ and $\mathrm{CsCl}$ lattices by their symmetry properties, and deduced the selection rules for optical transitions.

Thin layers of CuBr were prepared by sublimation in a high vacuum $\left(10^{-5}\right.$ to $\left.10^{-6} \mathrm{~mm} \mathrm{Hg}\right)$ at about $300^{\circ} \mathrm{C}$. The films, ranging in thickness from 100 and 10,000A, were deposited on quartz plates.

The room temperature absorption spectrum (Fig. 26) shows two sharp

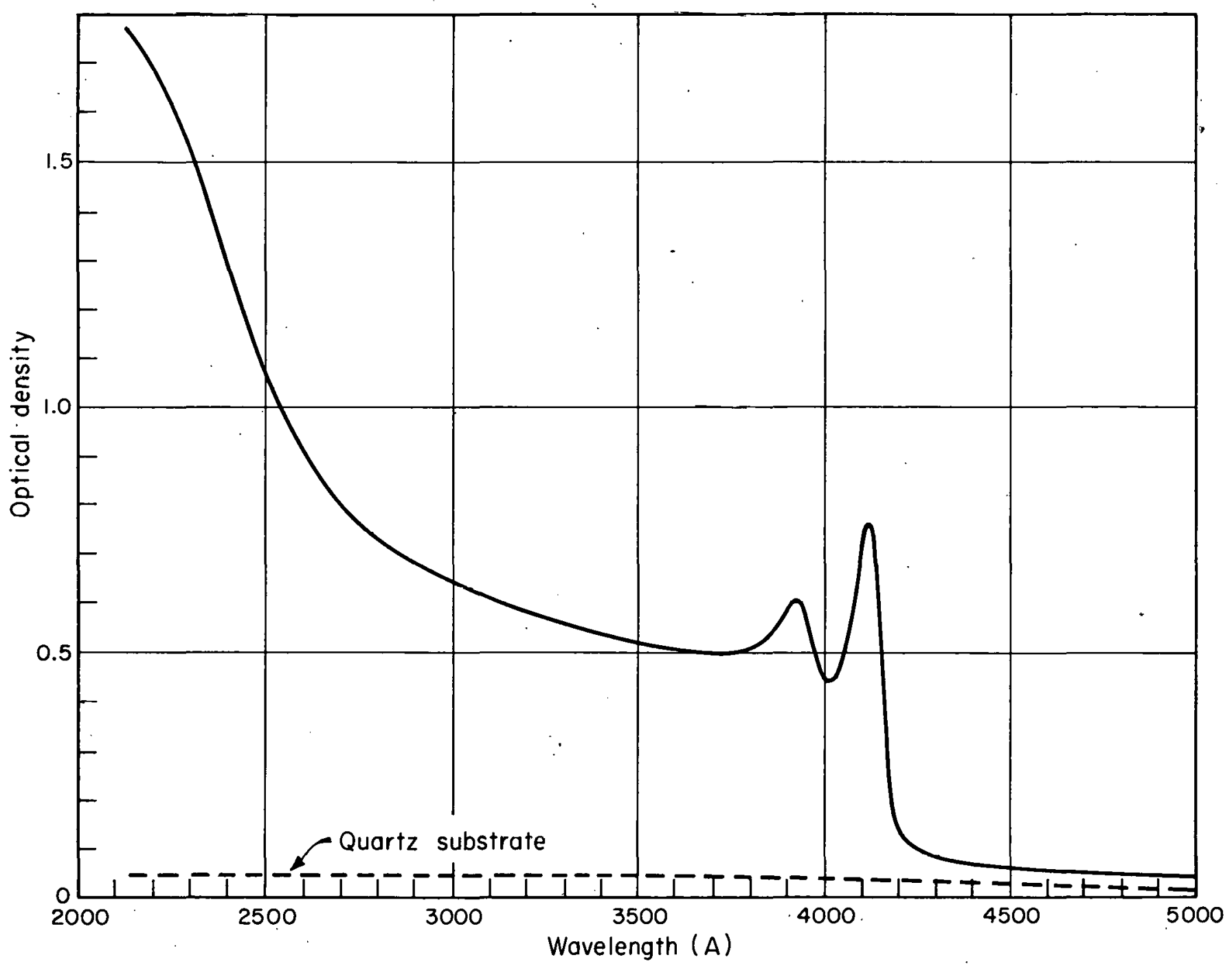

Fig. 26. Absorption of light by cuprous bromide film at room temperature. 
bands at 39.30 and $4130 \mathrm{~A}$, and a broad absorption around $2300 \mathrm{~A}$. The absorption constant between the bands (ca. $3800 \mathrm{~A})$ is of the order of $5 \times 10^{4} \mathrm{~cm}^{-1}$. At $-182^{\circ} \mathrm{C}$, the two bands (Fig. 27) shift toward longer wavelength by nearly 30A and are appreciably sharper. Weak additional bands can be discerned at $3818,4048,4113$, and $4193 \mathrm{~A}$ and also a weak band appears at. $2470 \mathrm{~A}$, in the tail of the ultraviolet absorption band, which is shifted toward shorter wave-

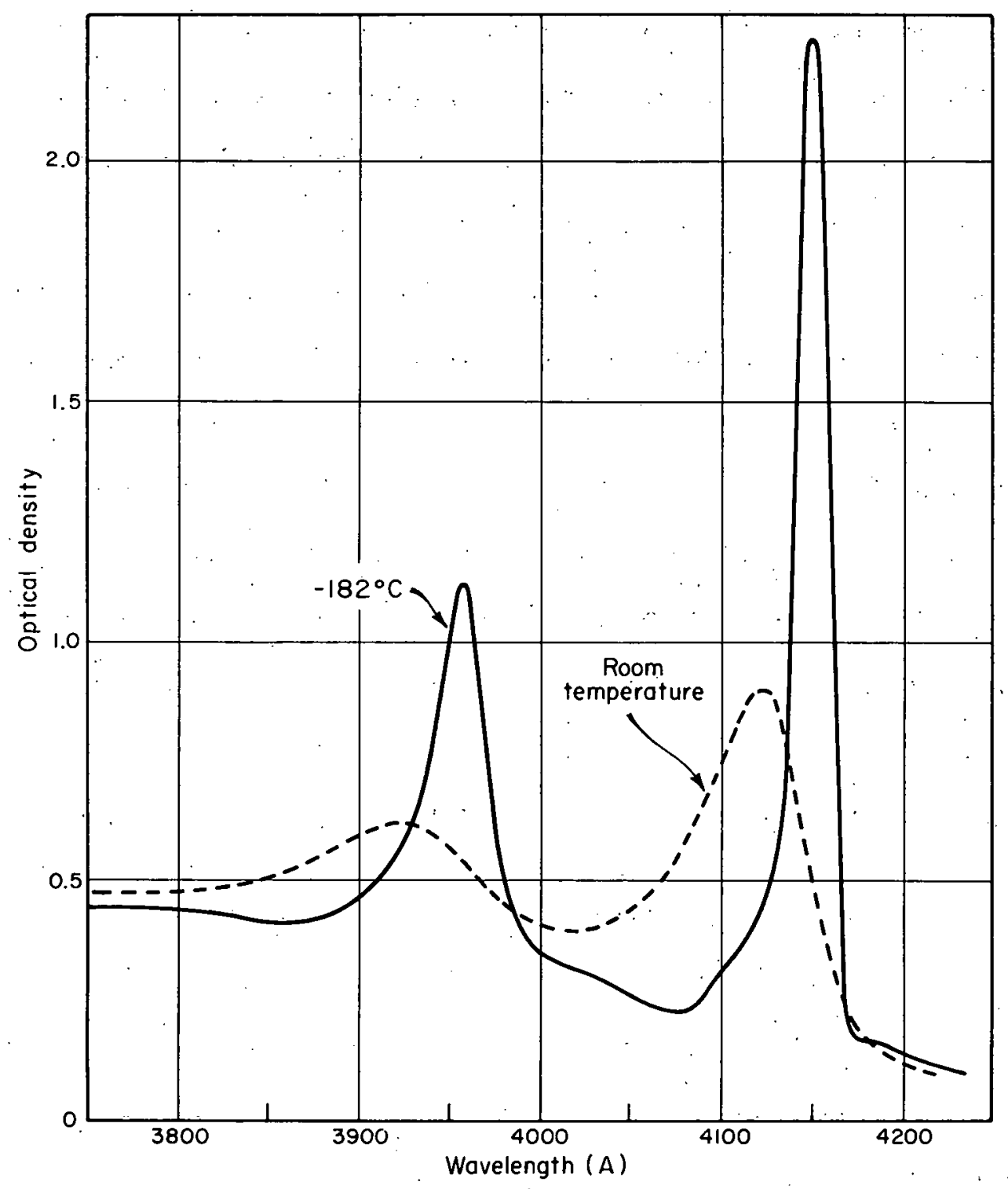

Fig. 27. Absorption of light by cuprous bromide film. 
length.

- A slightly thicker film at $-191^{\circ} \mathrm{C}$ indicates an additional band at about $4165 \mathrm{~A}$, so that the spectrum in the $4000 \mathrm{~A}$ range may be composed of two strong and probably five weak absorption bands. . A detailed investigation of the influence of temperature and magnetic field on the position and half width of the bands is now in progress.

A single crystal of $\mathrm{CuBr}$ has been grown by the Bridgman method, from commer cial cuprous bromide purified by sublimation in vacuo. The crystal has a somewhat milky appearance, probably due to the presence of the high-temperature hexagonal phase, and has a greenish tinge. It shows high conductivity at room temperature, is photosensitive and emits a strong red luminescence under' Wood's light at liquid $\mathrm{N}_{2}$ temperature. Small clear crystals obtained by sublimation do not emit in the red, but only in the violet.. The study is: continuing:

Following Overhauser's approach, we have derived the selection rules for exciton transition in an ionic crystal having the zinc-blende structure. Two optical transitions are allowed from the ground state to a singlet state, and 5 to a triplet state. The latter transitions, involving a change of the total spin of the system, are possible only through spin-orbit interaction and hence might have produced the weak absorption bands.

2. Optical and electrical properties of binary compounds of $\mathrm{II}_{\mathrm{b}}$ with $\underline{\mathrm{VI}_{\mathrm{b}} \text { elements }}$ (G. B. Wright).

At the start of a program of study of the optical and electrical properties of II-VI compounds, measurements were made on thin crystals of CdS.* The optical absorption edgè wàs studied at room temperature for different polarizations and an attempt was made to influence the edge with

\footnotetext{
* Kindly supplied by Dr. R: H: Bubé of $\mathrm{RCA}$.
} 
a magnetic field. The available magnetic field (ca. 10,000 gauss) was not. sufficiently strong and it is planned to extend the se measurements to higher: fields and lower temperatures:

Mercury selenide and telluride were prepared by reaction of the elements in evacuated Vycor tubes at $700^{\circ} \mathrm{C}$, and crystals were grown in a Bridgman furnace.

3. Electrical conductivity of magnetite (D. S. Tannhauser)

The great effect of oxygen concentration on the conductivity of magnetite mentioned in the last progress report was spurious since a phase transition to hematite had occurred.

At present we are investigating the influence of the iron-to-oxygen ratio and conductivity below room temperature, especially in the transition region at $-119^{\circ} \mathrm{C}$. The ratio $\mathrm{Fe}: \mathrm{O}$ is varied by annealing the samples at $1450^{\circ} \mathrm{C}$ under controlled $\mathrm{O}_{2}$ pressure until equilibrium is established and then quenching the samples. Magnetoresistance and Hall effect measurements on the se samples are planned.

4. Solid ionic conductors in electrochemical power sources (J. N. Mrgudich) It is known ${ }^{12)}$ that a thin layer of certain solid ionic conductors can"be used as the "electrolyte" of an electrochemical power.source. An example would be an array consisting of thin laminations of silver, solid silver iodide and manganese dioxide. The present status: of such solid electrolyte: "batteries." has been summarized by Shapiro. ${ }^{13)}$ The potential advantages over conventional liquid electrolyte batteries are ease of miniaturization, improvement in shelf-life, ruggedness, simplified production and, probably a wider

12) K. Lehovec and J. Broder, J. Electrochem. Soc. 101; 208 (19.94).

13). S. Shapiro; Proc. 11th Battery Conf., Asbury Park, N. J.., May, ,1957; p. 3. 
temperature range of operation. The most serious disadvantage of solid electrolyte systems developed to date is very high internal resistance, and therefore application is limited to the relatively few cases where current requirements are of the order of a few microamperes.

The purpose of the present study is to understand the factors causing the high internal resistance and, if possible, to eliminate them. At present we are concentrating on silver iodide. Three approaches suggest themselves:

1. Increase of the ionic conductivity by (a) enhanced surface conductivity; (b) doping and (c) by change of the crystal structure.

2. Study of barrier effects which.inhibit the free movement of silver ions from a silver surface into the silver halide and from the halide into the depolarizer.

3. Investigation of the complex ionic-electronic processes in the depolarizer which discharges the silver ion.

Work to date has highlighted the importance of the second approach. In the course of our measurements of ionic conductivity, we found that the use of amalgamated silver electrodes results in a sharp reduction of the polarization effects. Figure 28 shows the conventional time dependence of the specific conductivity of a pellet of compressed AgI powder between clean, polished. silver electrodes as compared to the much higher and nearly time-independent conductivity of the same pellet between silver electrodes amalgamated by momentary immersion in pure mercury. It appears that phenomena associated with electrode interfaces are probably the prime factors limiting the current output of present-day solid-electrolyte battery systems,

In connection with studies on the crystal structure of AgI we found that the hexagonal $\beta$ phase can be converted to the face-centered cubic $\gamma$ face by 


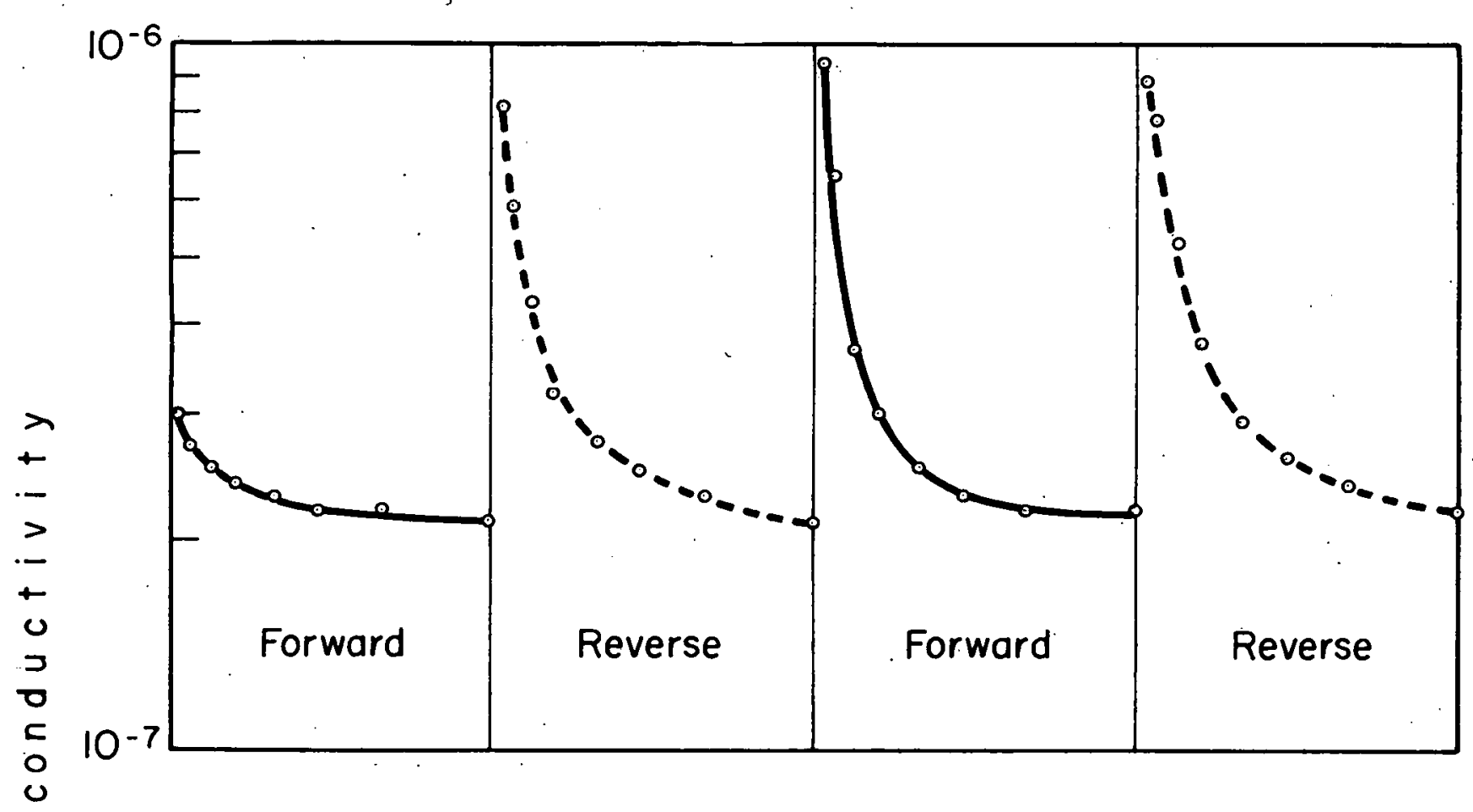

(a)

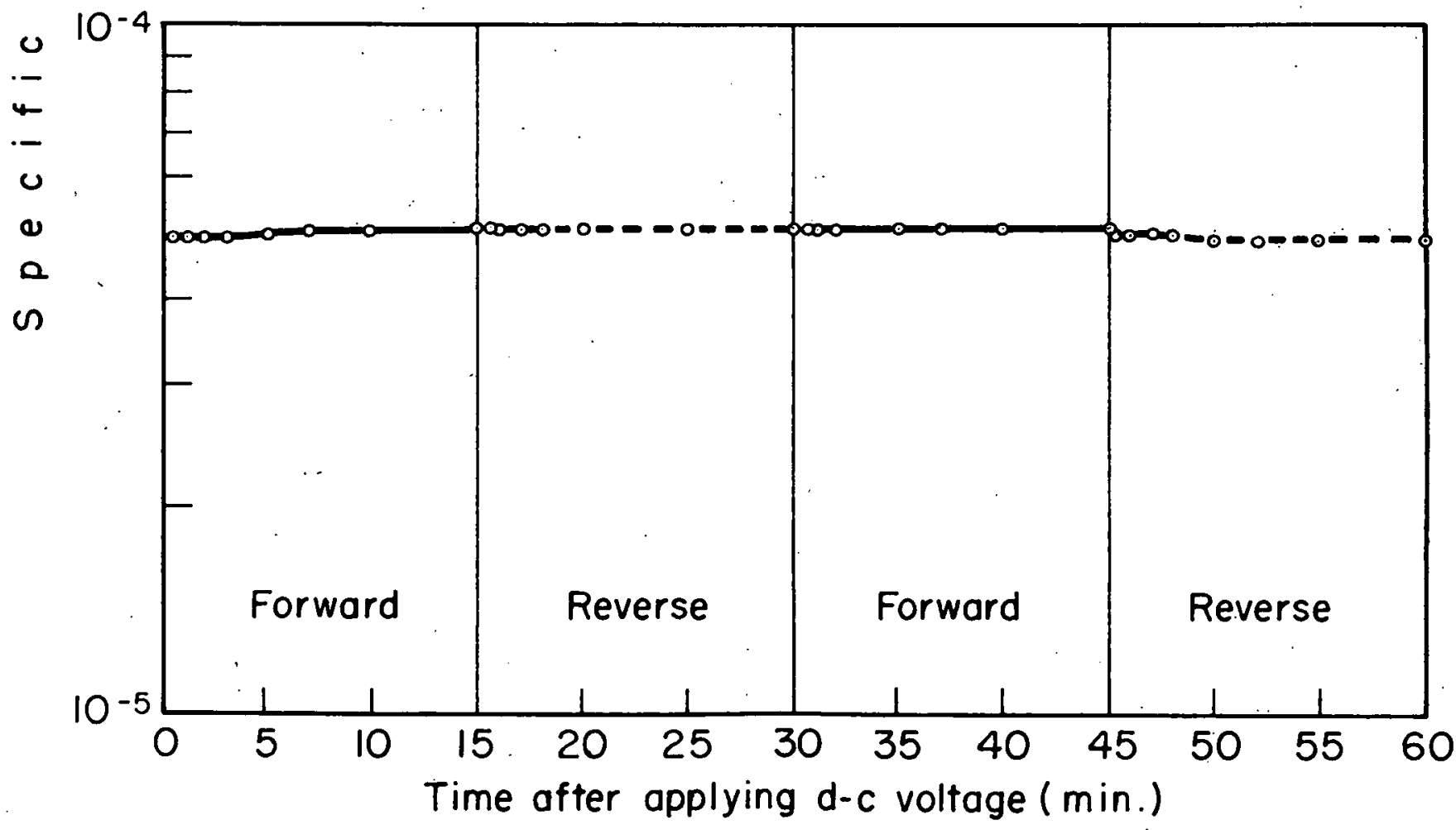

(b)

Fig. 28. Effect of electrodes on polarization in AgI:

(a) unamalgamated; (b) amalgamated. 
pressures of less than 10,000 psi at room temperature. Work on compressed powders and single crystals is continuing.

5. Hall-effect instrumentation (W. A. Navipour)

A low-temperature sample holder and a Dewar flask have been constructed in which the reservoir level can be adjusted independently of the level of the liquid bath (Fig. 29). A temperature control system has been tested and

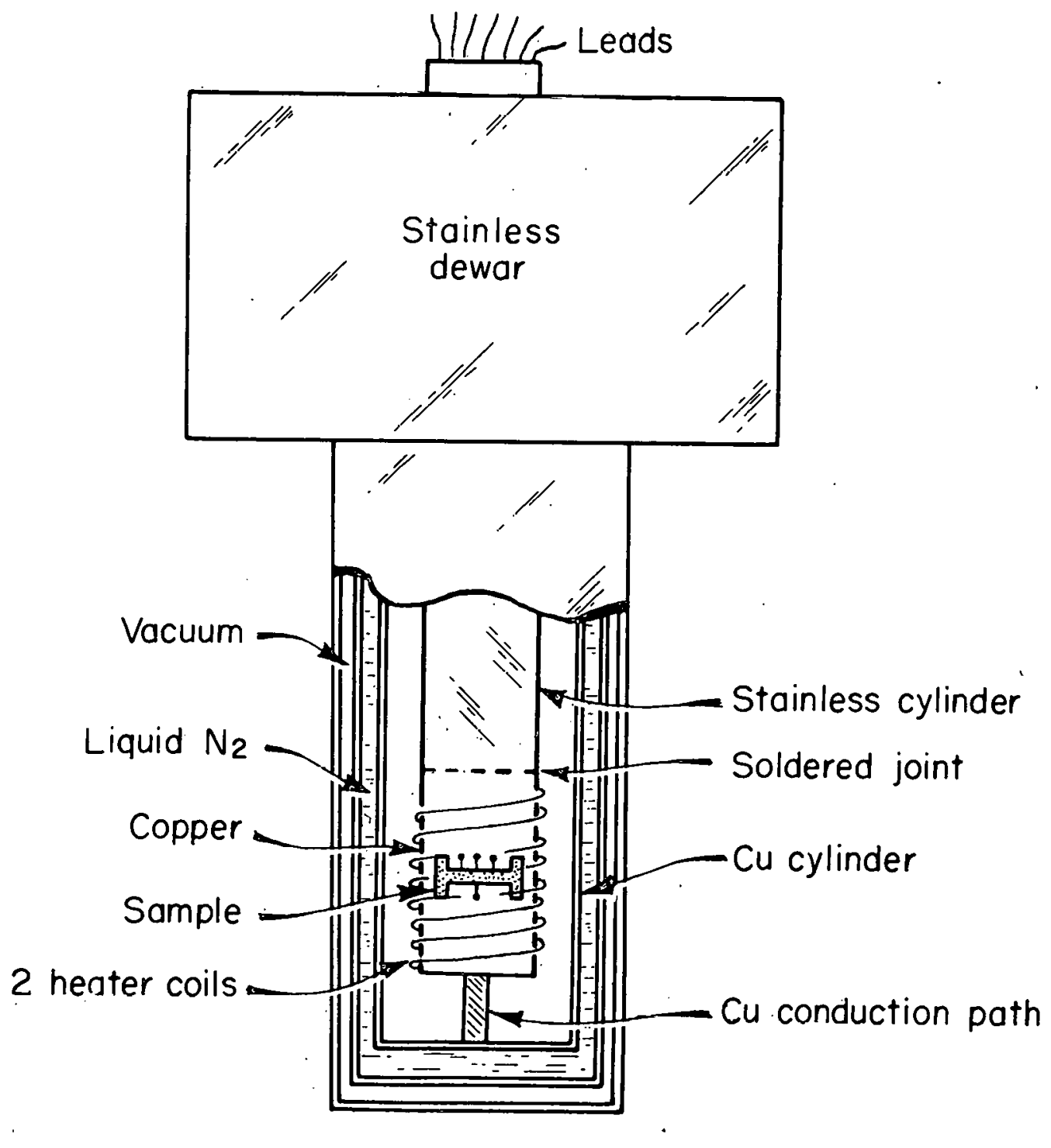

Fig. 29: Low-temperature flask and sample holder. 
should allow constancy to better than $0.1^{\circ} \mathrm{C}$. A Hall equipment for low-input impedance has been set up, trial runs on Ge have been made and improvements are being incorporated on the basis of these tests.

A high-impedance Hall equipment is in the design stage.

6. Hall-effect apparatus for low-impedance samples (D. S. Tannhauser)

The apparatus, which is a modified version of one described by Dauphineé and Mooser ${ }^{14)}$ and which uses as far as possible commercially available components; is being tested. It should permit currentless measurements of resistances and of $R_{H} B / h\left(R_{H}\right.$ is the Hall constant, $B$ the magnetic field, $h$ the thickness of the sample) from $10^{5} \Omega$ to $10^{-6} \Omega$ with one reading of a dial. The apparatus operates at $40 \mathrm{cps}$ and thus eliminates the influence of thermal EMF. Since two resistances are compared, current fluctuations and the quality of the electrodes is expected not to be critical.

\section{SINGLE CRYSTALS}

1. Survey (A. Smakula)

The purification of alkali halides by zone refining has been continued with the aim of establishing its ultimate performance. Thermal etching is proving to be a purposeful tool in the study of crystal imperfections and dissolution phenomena. The influence of crystallographic direction on crystal growth has been investigated by ionic conductivity measurements with interesting results. Large oriented single crystals of lead have been grown for neutron spectrometers. In connection with new color-center investigations the growing of crystals from solution has been started and earth-alkali fluoride crystals containing controlled amounts of additives will be grown.

14) T. M. Dauphineé and E. Mooser, Rev. Sci. Instr. 26, 660 (1955). 
2. Purification of alkali halides by zone refining (B. J. Wuensch)

$\mathrm{X}$-ray lattice constant measurements, precision density measurements and spectrochemical analysis of zone-refined potassiumi iodide, described in the preceding progress reports, indicate that zone refining can remove most of the impurities from alkali halides. To determine the efficiency of the process and to what extent crucible contamination limits the ultimate purity, 100-g samples of refined potassium iodide were prepared and one, five, ten, and twenty passes of zone refining were used. Analyses including conductivity measurements are in progress.

3. Thermal etching (A. Smakula and L. A. Krassner)

Chemical etching has long been used for surface studies of metals and in recent years very successfully for semiconductors. Chemical etching is not suitable for water-soluble crystals. Thermal etching, on the other hand, can be used in all cases, particularly for crystals with a high vapor pressure. The method is very simple: a crystal is heated in vacuo at a specified temperature and for a specified time. If the temperature is sufficiently. below the melting point, evaporation starts at certain points on the crystal and regular pits develop. In Fig. 30 is shown the structure of an etch pit obtained on a cleaved surface of potassium bromide at $500^{\circ} \mathrm{C}$ after $20-\mathrm{h}$. heating. The edges form somewhat distorted steps but the bottom of the inversed pyramid is quite smooth. One can conclude from the structure of the edges that the continuation of the evaporation from an etch pit is at the corners. Of several hundred pits inspected, not one gave an indication of spiral dislocations. Two peculiar effects have been observed: the density of the etch pits on surfaces cleaved parallel to the growing direction is ca. 10 times that on perpendicular faces and they appear only on the upper surfaces. These effects are under further study. 

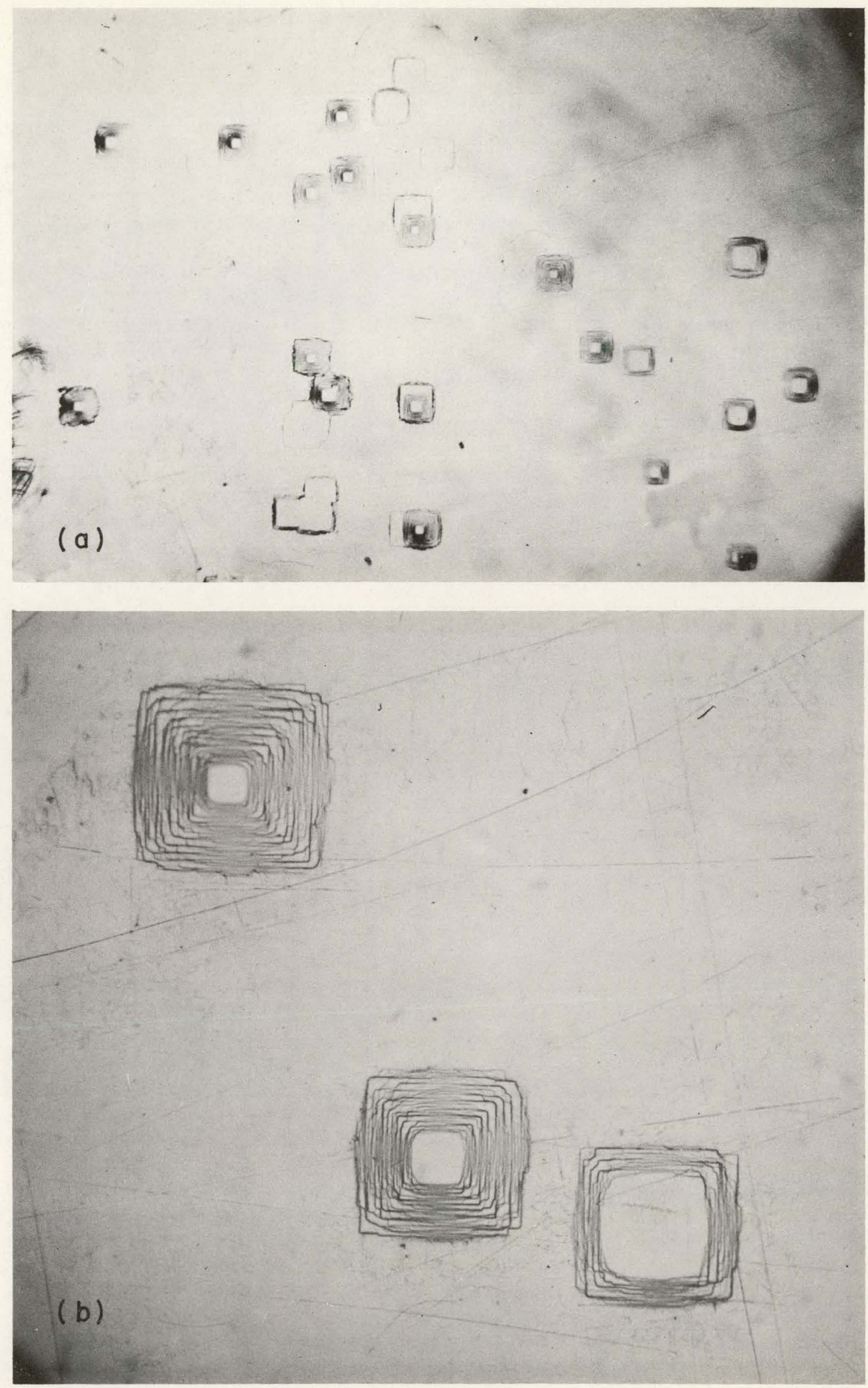

Fig. 30. Thermal etch pits on (100) face of potassium bromide: (a) distribution; (b) edge structure. 
4. Ionic conductivity of potassium bromide single crystals (V. Klemas)

A variety of methods (e.g., density, lattice constant, absorption, etc.) have been used for the study of crystal imperfections. Since each may reveal a different type of imperfection, the same crystal should be investigated by several methods and the results compared. In the work repeated here we measured the ionic conductivity as function of the crystal-growing direction.

Three $\mathrm{KBr}$ crystals were grown by the pulling method under exactly the same conditions but with the growth direction orientated in the [110] , [111] or [100] direction. Their ionic conductivity was then measured in the range from $100^{\circ}$ to $600^{\circ} \mathrm{C}$ (Fig. 31).

Growing in the $[111]$ and $[100]$ directions produces crystals with considerably lower conductivity than in $[110]$.

5. Orientated growing of large lead single crystals (H. Dachs and J. Kalnajs) Large single crystals of lead have now been grown for measurements in a neutron monochromator. The crystal cut had to be parallel to the (111) plane, hence the $[110]$ direction to make the best use of the material. For the orientated crystal growth, seeds of ca. $1.5 \times 0.5 \times 0.5$ in. were cut from smaller crystals grown without preferred orientation. They were grown from a c.p., filtered metal in small graphite crucibles in an evacuated glass vessel. A large covered crucible ( 2 in. dia. $x 13$ in.) was made of reactor-grade graphite. To mount the seed, a cavity was cut in the bottom of this crucible, and the seed with its (110) face up was cemented in the cavity with graphitepowder putty containing a small amount of sodium silicate binder. The temperature gradient was adjusted so that one half of the seed would be molten. Clean pieces of lead were gradually loaded into the crucible. Stirring of the melt 


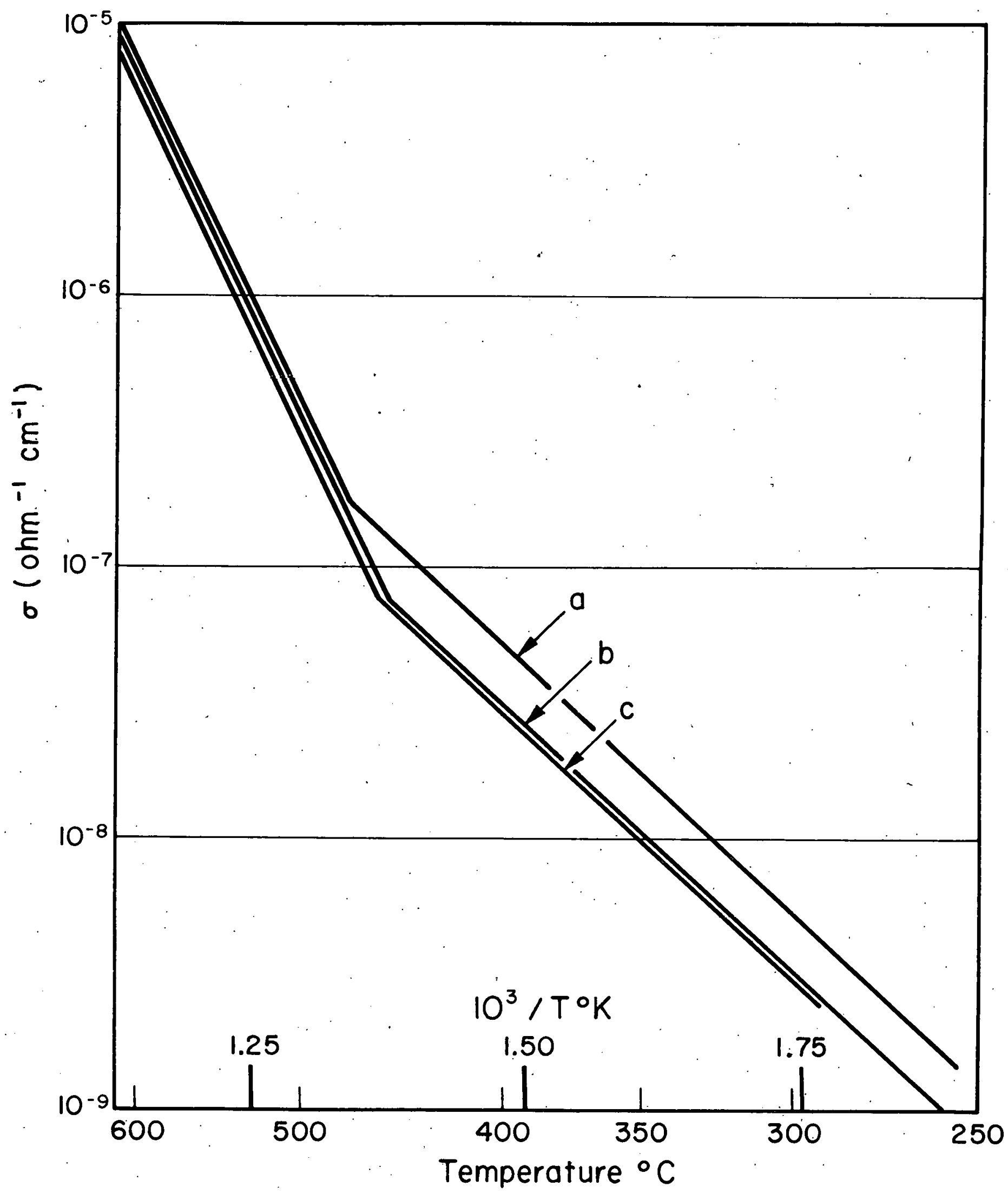

Fig. 31. Ionic conductivity of potassium bromide crystals grown in (a) [110]; (b) [111], (c) [100] direction. 
secured good contact of the molten metal with the seed, brought lead oxide to the surface, and released trapped air. Growing speeds of $9 \mathrm{~cm} / \mathrm{h}$. yielded in most cases $2 \times 11$ in. single crystals of cylindrical shape, and with nearly the proper orientation.

Molding of the soft lead crystal in a square block of self-setting plaster gave the necessary support and protection during cutting. Dilute nitric acid removed the damaged surface layers. The crystal was then embedded in plaster in a special holder and the orientation and mosaic structure was rechecked by $X$ rays. Finally, the crystal was cut on the milling machine to the precise angle desired.

\section{Color centers in cesium halides ( $P$. Avakian)}

Color centers in cesium halides have a short.lifetime at room temperature (a few minutes for $\mathrm{CsC1}$ ). Furthermore, $\mathrm{CsCl}$ changes from face- to body-centered cubic as the temperature is lowered (transition temperature, ca. $469^{\circ} \mathrm{C}$ ). Single crystals have therefure to be grown from solution, and since the material is also very expensive, not much work has been done on the cesium halides.

Equipment has been set up for crystal growing from solution, seed crystals have been obtained and the growing of large crystals is in progress.

7. Color centers in alkaline-earth fluorides (W. J. Scouler)

The absorption bands due to color centers in $\mathrm{CaF}_{2}$ have been measured by several investigators with inconsistent results. Most of the crystals used have been natural ones, but some were artificially grown from the melt. Color centers have generally been produced by $\mathrm{X}$ or cathode rays. The bands produced depended on the source of the samples and the methods of discoloration. Stockbarger ${ }^{15)}$ grew single crystals from the melt by the Bridgman

15) D. C. Stockbarger, Discussions Faraday Soc. 5, 294 (1949). 
temperature-gradient technique in graphite crucibles. The optical quality of his crystals compared well with that of the best natural crystals.

Since $\mathrm{CaF}_{2}$ melts around $1400^{\circ} \mathrm{C}$, the main problems have involved proper heat shielding, proper temperature measurement and control, and proper atmospheric control. We intend to use induction heating to grow single crystals by the Bridgman technique. Trial runs with various furnace designs are in progress.

\section{CERAMICS}

1. High-density ceramics (, . Ficononos)

Studies on ceramics as capacitor dielectrics necessitate the preparation of high-density specimens. The materials under study are high purity $\mathrm{TiO}_{2}, \mathrm{BaTiO}_{3}, \mathrm{SrTiO}_{3}, \mathrm{CaTiO}_{3}$; and $\mathrm{MgTiO}_{3} \cdot{ }^{*}$ Initial preparation consists of grinding the powder in a mullite mortar for 30 min., adding Carbowax No. 4000 in trichlorethyelene, pressing in a steel die at 5000 psi and repressing in a hydrostatic chamber at 30,000 psi. Firing at $1375^{\circ} \mathrm{C}_{\text {for }} \mathrm{BaTiO}_{3}$ and $\mathrm{SrTiO}_{3}$, and $1400^{\circ} \mathrm{C}$ for $\mathrm{TiO}_{2}, \mathrm{CaTiO}_{3}$, and $\mathrm{MgTiO}_{3}$ in a flowing-air furnace atmosphere resulted in material having the densities shown in Table 1.

Table 1. Density of fired ceramic specimens.

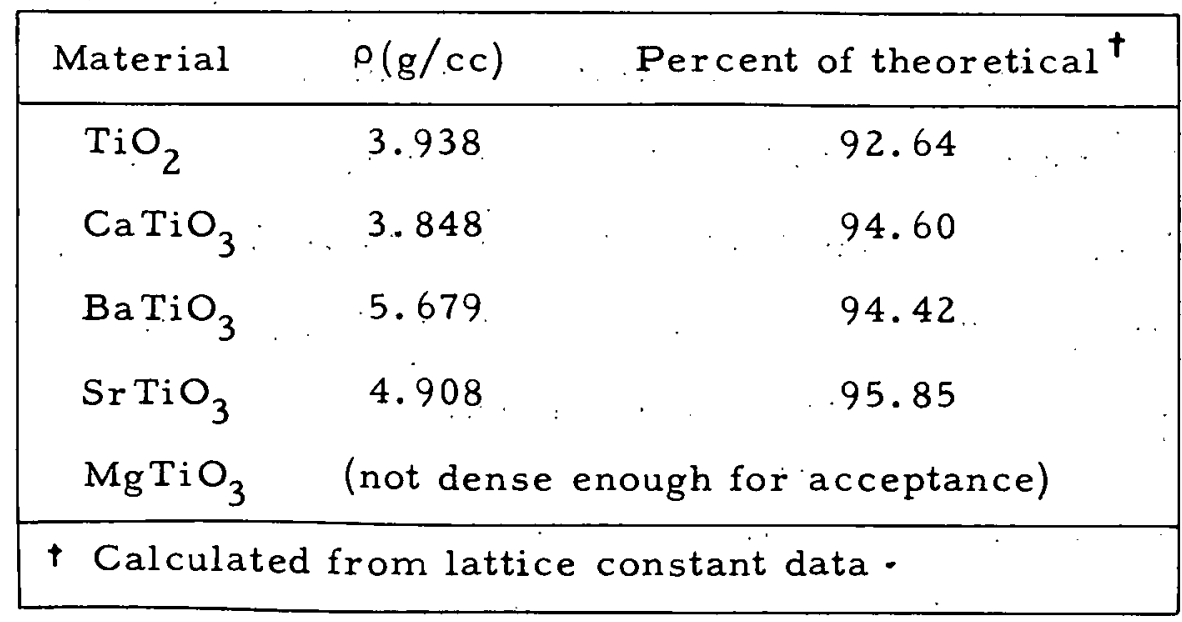

\footnotetext{
* National Lead Co., Niagara Falls, N. Y.
} 
Work is continuing to determine optimum conditions for achieving higher density.

2. High-pressure firing (T.R. Clevenger)

The high-pressure equipment mentioned in Progress Report No. XXI has been assembled and the necessary heat-controlling and cooling apparatus installed. Test runs and work has been started on a detailed investigation of $\mathrm{BaTiO}_{3}$ ceramics as function of particle size.

3. Magnetic exchange interactions (J. S. Waugh)

The system La $1-\mathrm{Ba}_{\mathbf{x}} \mathrm{FeO}_{3}$ is being studied in detail for values of $\mathrm{x}$ up to the solid-s,olution limit to better understand the reported anomaly of nonferromagnetism in this system (the same systems of cobalites and manganites are ferromagnetic). Measurements of magnetization, susceptibility, and electronic conductivity should yield valuable information about the exchange mechanism in the se ionic solids.

The system La ${ }_{1-\mathbf{x}} \mathrm{Ba}_{\mathbf{x}} \mathrm{TiO}_{3}$ is being studied in an identical manner. By varying the cation which occupies the octahedral site (these systems are perovskites), the role of its electronic configuration can be evaluated. Other nonoxide systems are also to be investigated. By studying both oxide and nonoxide systems, the role of the oxygen ion can be more clearly understood.

The present status of the work is (1) the systems $\mathrm{La}_{1-\mathrm{x}} \mathrm{Ba}_{\mathrm{x}} \mathrm{FeO}_{3}$ and $\mathrm{La}_{1-\mathrm{x}} \mathrm{Ba}_{\mathbf{x}} \mathrm{TiO}_{3}$ have been prepared and identified by $\mathrm{X}$ rays; (2) the construction of equipment necessary for measurement of magnetization and conductivity from liquid helium to $775^{\circ} \mathrm{K}$ is near completion.

4. Modified atomizing-burner operation (G. Economos)

The atomizing burner is a promising tool for the preparation of finegrained, highly reactive oxide powders over a wide compositional range 
(cf. Progress Report XXI). Several difficulties appeared in operation: the extinction of the flame when the combustion chamber is cold; the piling up of material at the end of the combustion chamber; and the "blow-back" at the nozzle.

The first difficulty was overcome by replacing the external gas flame with a pair of spark plugs placed $120^{\circ}$ apart near the bottom of the combustion chamber and 2 in. from the nozzle; a 10,000 v oil-burner ignition transformer supplies the spark. Early condensation at the cool end of the combustion chamber was eliminated by shortening the chamber from 18 to 14 in. so that its entire length is now heated to high temperature. Avoidance of the blowback called for increase of the gas volume. A perforated ring was placed at the periphery of the nozzle through which an air blast could be directed into the combustion chamber. This provides excess oxygen for a more complete combustion, and an increased gas volume for carrying the products of combustion into the cyclone chamber.

These modifications have led to a marked improvement in performance. Two remaining difficulties are the instability of the alcohol solutions and the loss of about 50 percent of the fine material with the exhaust gases. 